\title{
Methicillin-resistant Staphylococcus aureus alters cell wall glycosylation to evade immunity
}

David Gerlach ${ }^{1,2,13}$, Yinglan Guo ${ }^{3,13}$, Cristina De Castro ${ }^{4}$, Sun-Hwa Kim ${ }^{5}$, Katja Schlatterer ${ }^{1,2}$, Fei-Fei Xu' ${ }^{6}$, Claney Pereira ${ }^{6}$, Peter H. Seeberger ${ }^{6}$, Sara Ali ${ }^{7}$, Jeroen Codée ${ }^{7}$, Wanchat Sirisarn ${ }^{8}$, Berit Schulte ${ }^{2,9}$, Christiane Wolz ${ }^{2,9}$, Jesper Larsen ${ }^{10}$, Antonio Molinaro ${ }^{11}$, Bok Luel Lee ${ }^{5}$, Guoqing Xia ${ }^{8}$, Thilo Stehle ${ }^{3,12,14 *} \&$ Andreas Peschel ${ }^{1,2,14 *}$

Methicillin-resistant Staphylococcus aureus (MRSA) is a frequent cause of difficult-to-treat, often fatal infections in humans ${ }^{1,2}$. Most humans have antibodies against $S$. aureus, but these are highly variable and often not protective in immunocompromised patients ${ }^{3}$. Previous vaccine development programs have not been successful ${ }^{4}$. A large percentage of human antibodies against $S$. aureus target wall teichoic acid (WTA), a ribitol-phosphate (RboP) surface polymer modified with $N$-acetylglucosamine $(\mathrm{GlcNAc})^{5,6}$. It is currently unknown whether the immune evasion capacities of MRSA are due to variation of dominant surface epitopes such as those associated with WTA. Here we show that a considerable proportion of the prominent healthcare-associated and livestock-associated MRSA clones CC5 and CC398, respectively, contain prophages that encode an alternative WTA glycosyltransferase. This enzyme, TarP, transfers GlcNAc to a different hydroxyl group of the WTA RboP than the standard enzyme $\operatorname{TarS}^{7}$, with important consequences for immune recognition. TarP-glycosylated WTA elicits 7.5-40-fold lower levels of immunoglobulin G in mice than TarS-modified WTA. Consistent with this, human sera contained only low levels of antibodies against TarP-modified WTA. Notably, mice immunized with TarS-modified WTA were not protected against infection with $\operatorname{tar} P$-expressing MRSA, indicating that TarP is crucial for the capacity of $S$. aureus to evade host defences. High-resolution structural analyses of TarP bound to WTA components and uridine diphosphate GlcNAc (UDPGlcNAc) explain the mechanism of altered RboP glycosylation and form a template for targeted inhibition of TarP. Our study reveals an immune evasion strategy of $S$. aureus based on averting the immunogenicity of its dominant glycoantigen WTA. These results will help with the identification of invariant $S$. aureus vaccine antigens and may enable the development of TarP inhibitors as a new strategy for rendering MRSA susceptible to human host defences.

Novel prevention and treatment strategies against major antibioticresistant pathogens such as MRSA are urgently needed but are not within reach because some of the most critical virulence strategies of these pathogens are not understood ${ }^{8}$. The pathogenic potential of prominent healthcare-associated (HA)-MRSA and recently emerged livestock-associated (LA)-MRSA strains is thought to rely on particularly effective immune evasion strategies, whereas communityassociated (CA)-MRSA strains often produce more aggressive toxins ${ }^{1,2}$. Most humans have high overall levels of antibodies against $S$. aureus as a consequence of preceding infections, but antibody titres differ strongly for specific antigens and are often not protective in immunocompromised patients, for reasons that are not clear ${ }^{3}$. A large percentage of human antibodies against $S$. aureus is directed against WTA ${ }^{5,9,10}$, which is largely invariant. However, some $S$. aureus lineages produce altered WTA, which modulates, for instance, phage susceptibility ${ }^{7,11}$.

To investigate whether some prevalent $S$. aureus lineages use additional WTA-targeted strategies to increase their fitness and pathogenicity, we screened $S$. aureus genomes for potential additional paralogues of WTA biosynthesis genes. We found three $S$. aureus prophages that encoded a protein, TarP, that has $27 \%$ identity to the WTA- $\beta$-GlcNAc

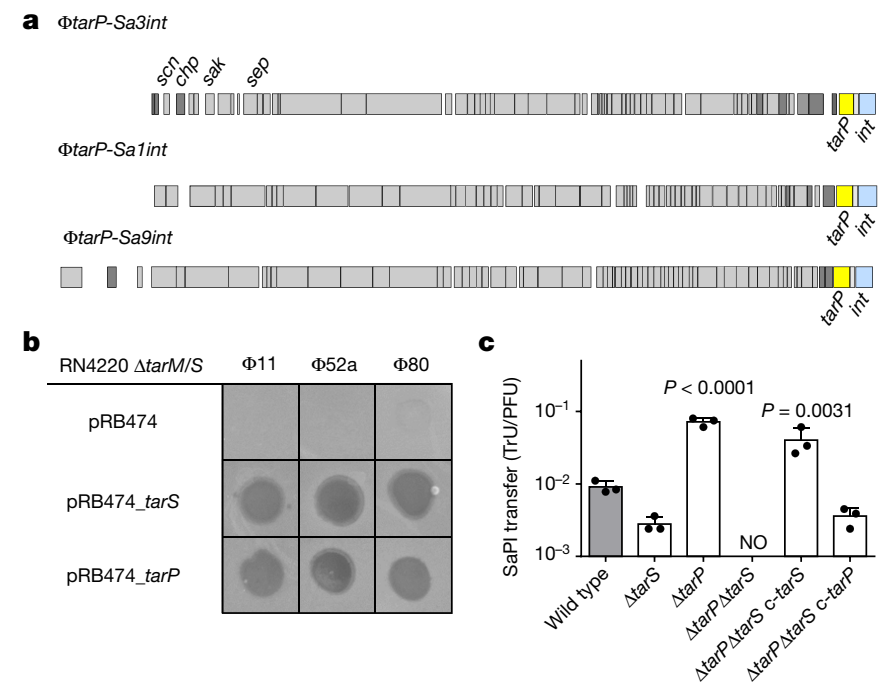

Fig. 1 The phage-encoded TarP can replace the housekeeping WTA $\boldsymbol{\beta}$-GlcNAc transferase TarS. a, TarP is encoded next to different integrase types (int gene) in prophages $\varphi$ tarP-Sa3int (with immune evasion cluster scn, chp, sak, sep), found in HA-MRSA, and $\varphi$ tarP-Salint and $\varphi$ tarP-Sa9int, identified in LA-MRSA. TarP variants in $\varphi$ tarP-Salint and $\varphi$ tarP-Sa9int differed from TarP in $\varphi$ tarP-Sa3int in one amino acid each (I8M and D296N, respectively). Both residues are distant from the catalytic centre. b, Complementation of $S$. aureus RN4420 $\Delta$ tarM/S with either $\operatorname{tar} S$ or $\operatorname{tar} P$ restores susceptibility to infection by WTA GlcNAcbinding siphophages, as indicated by plaque formation on bacterial lawns. Data shown are representative of three independent experiments. c, $\operatorname{tar} P$ expression reduces siphophage $\Phi 11$-mediated transfer of SaPIbov in N315. Values indicate the ratio of transduction units ( $\mathrm{TrU}$ ) to plaque-forming units (PFU) given as mean \pm s.d. of three independent experiments. Statistical significances when compared to wild type were calculated by one-way ANOVA with Dunnett's post-test (two-sided) and significant $P$ values $(P \leq 0.05)$ are indicated. NO (none obtained) indicates no obtained transductants.

\footnotetext{
${ }^{1}$ Interfaculty Institute of Microbiology and Infection Medicine, Infection Biology, University of Tübingen, Tübingen, Germany. ${ }^{2}$ German Centre for Infection Research (DZIF), Partner Site Tübingen,

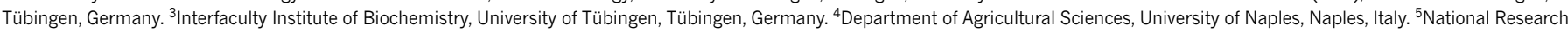
Laboratory of Defense Proteins, College of Pharmacy, Pusan National University, Pusan, South Korea. ${ }^{6}$ Max-Planck-Institute for Colloids and Interfaces, Potsdam, Germany. ${ }^{7}$ Leiden Institute of Chemistry, Leiden University, Leiden, The Netherlands. ${ }^{8}$ Lydia Becker Institute of Immunology and Inflammation, Division of Infection, Immunity and Respiratory Medicine, Faculty of Biology, Medicine and Health, University of Manchester, Manchester Academic Health Science Centre, Manchester, UK. 9 Interfaculty Institute of Microbiology and Infection Medicine, Medical Microbiology, University of Tübingen, Tübingen, Germany. ${ }^{10}$ Bacteria, Parasites and Fungi, Statens Serum Institut, Copenhagen, Denmark. ${ }^{11}$ Department of Chemical Sciences, University of Naples, Naples, Italy. ${ }^{12}$ Vanderbilt University School of Medicine, Nashville, TN, USA. ${ }^{13}$ These authors contributed equally: David Gerlach, Yinglan Guo. ${ }^{14}$ These authors jointly supervised this work: Thilo Stehle, Andreas Peschel. *e-mail: thilo.stehle@uni-tuebingen.de; andreas.peschel@uni-tuebingen.de
} 

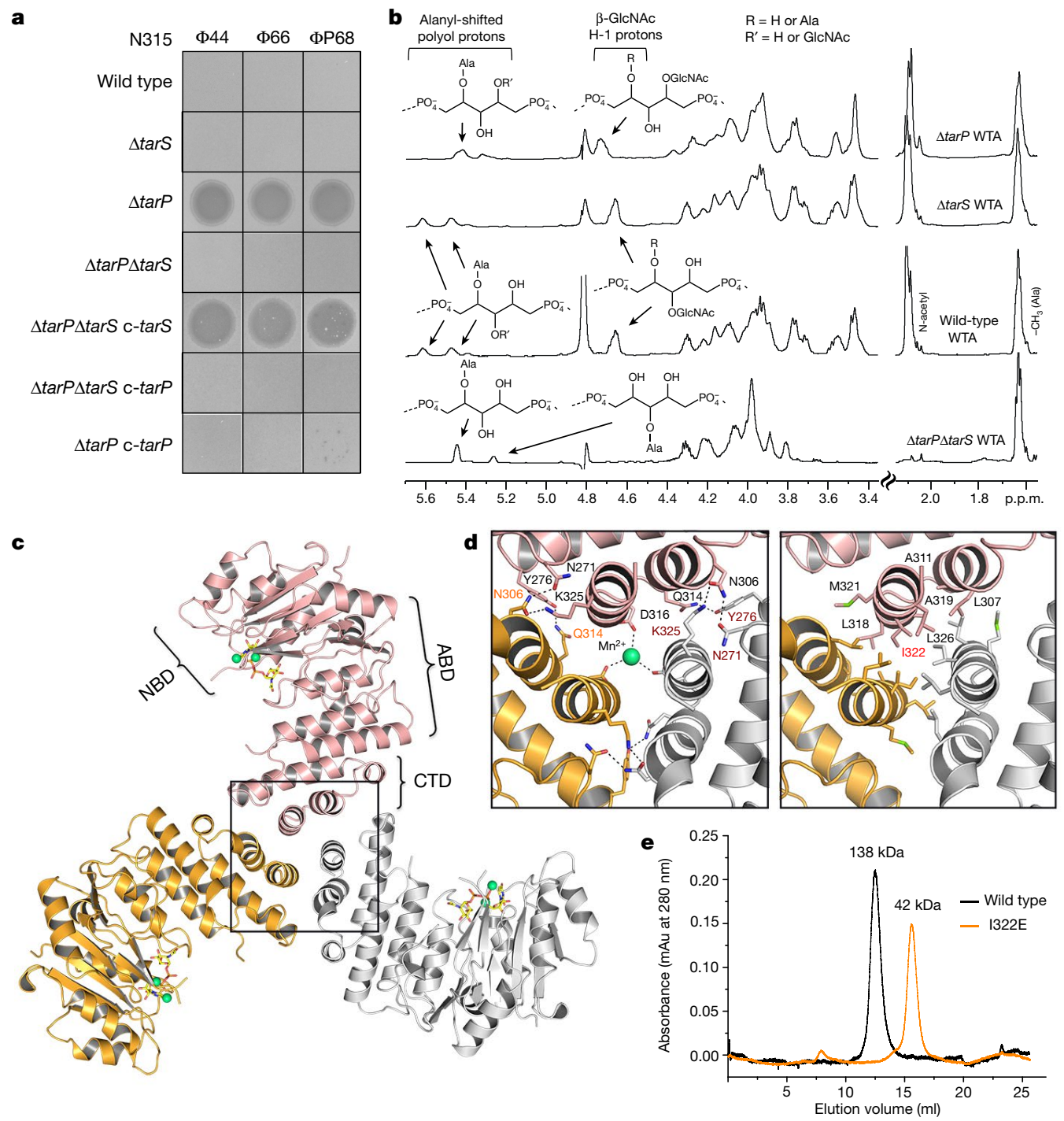

Fig. 2 TarP protects $\mathrm{N} 315$ from podophage infection by alternative glycosylation of WTA at RboP C3. a, Expression of tarP renders N315 resistant to podophages. Representative data from three independent experiments are shown. $\mathbf{b},{ }^{1} \mathrm{H}$ NMR spectra reveal different ribitol hydroxyl glycosylation of N315 WTA by TarS (C4) or TarP (C3). The RboP units with attached GlcNAc are depicted above the corresponding proton resonances. Representative data from three experiments are shown. In-depth description of the structural motifs identified in the spectra is given in the Supplementary Information. c, Crystal structure of TarP homotrimer (pink, orange, grey) bound to UDP-GlcNAc (yellow) and two $\mathrm{Mn}^{2+}$ ions (lime green). The nucleotide-binding domain (NBD), acceptor-

transferase $\operatorname{TarS}^{7}$ (Fig. 1a). $\operatorname{tar} P$ was found exclusively in isolates of the prominent HA-MRSA CC $5^{12}$, on a prophage that also encoded the $s c n, c h p$ and sak immune evasion genes ${ }^{13}$, and on two other prophages in the emerging LA-MRSAs CC $398^{14}$ and $\mathrm{CC}^{15}$. All tarP-harbouring genomes also contained tarS.

When tarP from CC5 HA-MRSA strain N315 was expressed in a WTA glycosylation-deficient mutant of laboratory strain RN42207, it restored WTA glycosylation (Extended Data Fig. 1a) and susceptibility to siphophages, which need RboP WTA GlcNAc as a binding motif $^{16}$ (Fig. 1b). The presence of $\beta$-GlcNAc on WTA is essential for full $\beta$-lactam resistance in MRSA strains ${ }^{7}$. When $\operatorname{tar} P$ was expressed in a WTA glycosylation-deficient mutant of CA-MRSA strain MW2 (CC1), it restored full oxacillin resistance (Extended Data Fig. 1b), confirming that $\operatorname{tar} P$ can replace $\operatorname{tar} S$ in several key interactions.

The expression of TarP led to susceptibility to siphophages, albeit to a lower extent than TarS (Extended Data Fig. 1c), although TarP did not binding domain $(\mathrm{ABD})$, and C-terminal trimerization domain (CTD) of the pink monomer are labelled. d, Views into the trimer interface (boxed in c). Left, polar interactions. Hydrogen bonds and salt bridges are shown as black dashed lines. The $\mathrm{Mn}^{2+}$ is $2.1 \AA$ from each Asp316 carboxylate. Right, hydrophobic interactions, with the mutated residue Ile322 highlighted in red. e, Size-exclusion chromatography elution profiles. Based on calibration of the column, the TarP wild-type and I322E mutant proteins have estimated molecular weights of $138 \mathrm{kDa}(n=8)$ and $42 \mathrm{kDa}$ $(n=3)$, respectively, in agreement with the calculated molecular weights of $120 \mathrm{kDa}$ for a TarP trimer and $40 \mathrm{kDa}$ for monomeric TarP.

incorporate less GlcNAc into WTA than TarS (Extended Data Fig. 1d, Supplementary Table 3). Similarly, the siphophage-mediated horizontal transfer of an $S$. aureus pathogenicity island was reduced about tenfold in $S$. aureus N315 expressing tarP, compared to the same strain expressing only tarS (Fig. 1c), suggesting that TarP and TarS glycosylate WTA differently. Notably, N315 was resistant to podophages, but inactivation of $\operatorname{tar} P$ (but not of $\operatorname{tar} S$ ) rendered it susceptible to podophages (Fig. 2a). We analysed the overall effect of tarP on podophage susceptibility patterns in 90 clinical CC5 and CC398 isolates and found that none of the $\operatorname{tar} P$-containing strains, but all of the $\operatorname{tar} P$-lacking strains, were susceptible to podophages (Extended Data Table 1). Thus, TarP causes podophage resistance and TarP-mediated modification of WTA is distinct from that mediated by TarS. Nuclear magnetic resonance (NMR) analyses revealed that both TarP and TarS add GlcNAc to WTA in the $\beta$-configuration. However, the attachment site in RboP differs: TarS glycosylates the C4 position ${ }^{17}$ whereas TarP attaches GlcNAc 

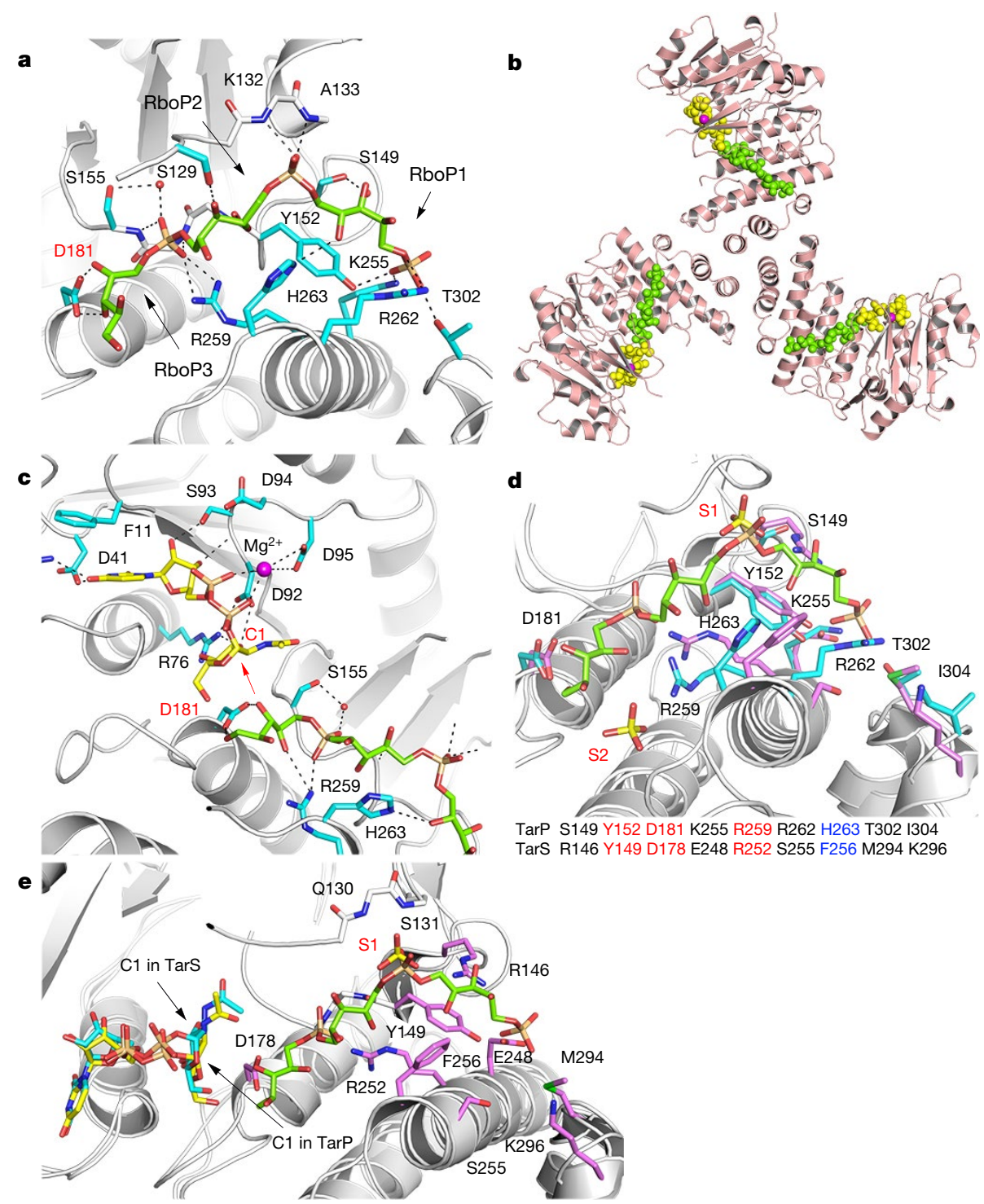

to C3 (Fig. 2b, Extended Data Fig. 2, Supplementary Table 2). This difference may be crucial for impairing phage infection. Moreover, NMR analysis revealed that TarP is dominant over TarS because in N315, which bears both genes, GlcNAc was almost exclusively attached to RboP C3 (Fig. 2b).

We solved the TarP structure at high resolution to elucidate how TarP generates a different glycosylation product from TarS. Like TarS ${ }^{18}$, TarP forms stable homotrimers, but it uses a different trimerization strategy because it lacks the C-terminal trimerization domain found in TarS (Fig. 2c, Extended Data Fig. 3). Instead, hydrophobic and polar interactions of a small helical C-terminal domain generate the TarP trimer (Fig. 2d, e). WTA polymers comprising three or six RboP repeating units (3RboP or $6 \mathrm{RboP}-\left(\mathrm{CH}_{2}\right)_{6} \mathrm{NH}_{2}$, respectively) were synthesized and used for soaking TarP crystals (Supplementary Information Fig. 2, 3), yielding the first protein structure visualizing the binding of a WTAbased polymer (Fig. 3, Extended Data Fig. 4). In the ternary complex TarP-UDP-GlcNAc-3RboP, the distance between the C3-hydroxyl of the third unit of $3 \mathrm{RboP}(\mathrm{RboP} 3$ ) and the anomeric $\mathrm{C} 1$ of GlcNAc is 4.2 $\AA$. Furthermore, at $3.1 \AA$, Asp 181 is well within hydrogen bonding distance of the C3-hydroxyl of RboP3. The observed distances and geometry nicely explain the unusual glycosylation of WTA at the C3-hydroxyl. We propose that TarP uses a direct $\mathrm{S}_{\mathrm{N}} 2$-like glycosyltransferase reaction, as discussed for other inverting GT-A fold enzymes ${ }^{19,20}$. In this mechanism, Asp181 would act as the catalytic base, deprotonating the C3-hydroxyl on RboP3 and enabling a nucleophilic attack on
Fig. 3 Interactions of TarP with UDPGlcNAc and D-ribitol-5-phosphate trimer (3RboP), and comparison of polyRboP binding sites of TarP and TarS. a, 3RboP binding site in the TarP-3RboP complex, with key amino acids shown (cyan). Asp181 is highlighted in red. The ribitol of $3 \mathrm{RboP}$ is coloured green and D-ribitol-5-phosphate units 1, 2 and 3 (RboP1, RboP2, and RboP3) are labelled. Hydrogen bonds and salt bridges are shown as black dashed lines. b, Ternary complex of TarP with UDP-GlcNAc and 3RboP. UDP-GlcNAc, $\mathrm{Mg}^{2+}$ and $3 \mathrm{RboP}$ are shown as full-atom models coloured yellow, magenta, and green, respectively. c, View into the active site of TarP. C1 of UDP-GlcNAc and Asp181 are highlighted with red labels. The arrow indicates how the C3-hydroxyl in RboP3 could nucleophilically attack GlcNAc C1. d, Comparison of the polyRboP-binding site of TarP with the corresponding region in TarS. Residues of TarP and 3RboP are coloured as in a. TarS residues are coloured violet and the two sulfates are labelled S1 and S2. Only residues of TarP are labelled, for clarity. Key TarP and TarS residues lining the polyRboP-binding site are shown at the bottom, with three identical (red) and one conserved amino acids (blue). e, Superposition of UDP-GlcNAc-bound TarS with the ternary TarP-UDP-GlcNAc-3RboP complex. UDP-GlcNAc and 3RboP bound to TarP are coloured as in $\mathbf{b}$, whereas UDPGlcNAc bound to TarS is coloured in cyan. Only the TarS residues are shown (coloured as in d), for clarity. The arrows indicate the $\mathrm{C} 1$ positions of UDP-GlcNAc in TarP and TarS. the GlcNAc C1, thus yielding a $\beta-O-G l c N A c y l a t e d ~ p o l y R b o P$ (Fig. $3 c$ ). Mutagenesis of Asp181 to alanine rendered TarP inactive, supporting this putative mechanism (Extended Data Table 2).

The ternary structure of TarP-UDP-GlcNAc-3RboP allows us to predict how polyRboP binds to the homologous TarS enzyme. Three residues that are critical for binding and catalysis (including Asp181) are identical in TarP and TarS, while five other residues differ ${ }^{18}$ (Fig. 3d). Lys255 and Arg262, for instance, which interact electrostatically with a WTA phosphate group in TarP, are replaced with Glu248 and Ser255, respectively, in TarS, which may lead to reduced affinity for WTA and might explain why TarP is dominant over TarS in vivo. On the basis of the location of UDP-GlcNAc, the identical Tyr149, Asp178 and Arg252 side chains, the conserved aromatic side chain of Phe256, and a site that contains a bound sulfate ion from the crystallization solution (S1) and probably binds phosphate in TarS (Fig. 3e), the polyRboP chain would be shifted to the upper right, and the relative position of RboP units in the binding site would be altered in TarS. Such an altered binding mode would move the C4-hydroxyl of the target RboP towards $\mathrm{C} 1$ of GlcNAc, allowing TarS to glycosylate at the C4 position.

$S$. aureus WTA is a dominant antigen for adaptive immune responses ${ }^{5,9}$. The observation that the position of GlcNAc on RboP had a profound impact on binding by podophage receptors raises the question of whether human antibodies also discriminate between the two isomeric polymers and whether MRSA clones use TarP to subvert immune recognition. We analysed several human antibody 
a

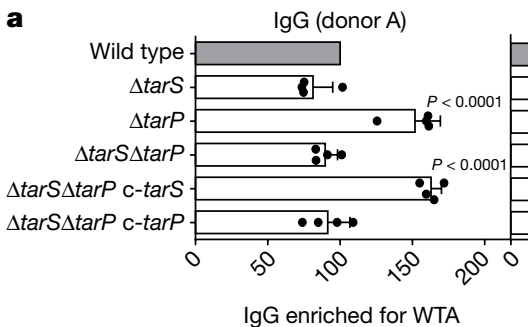

$\operatorname{lgG}($ donor B)

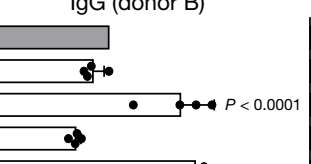

•? $P<0.000$

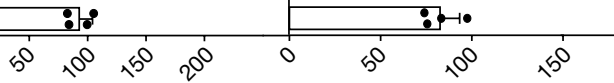

Pooled IgG (Abcam)

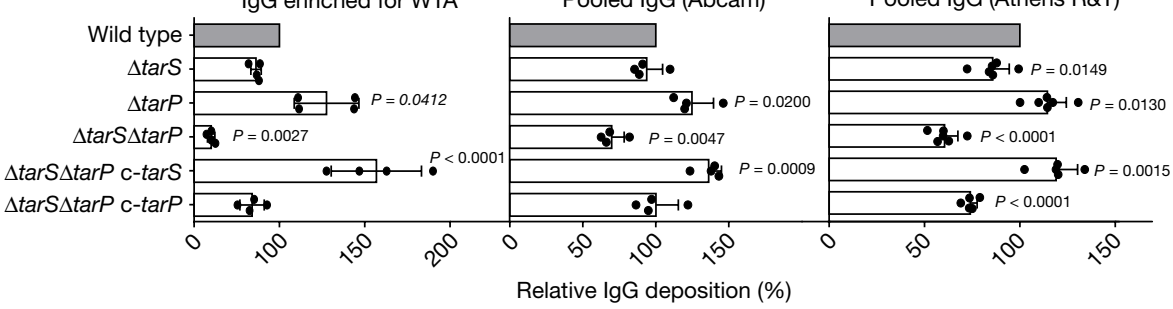

b

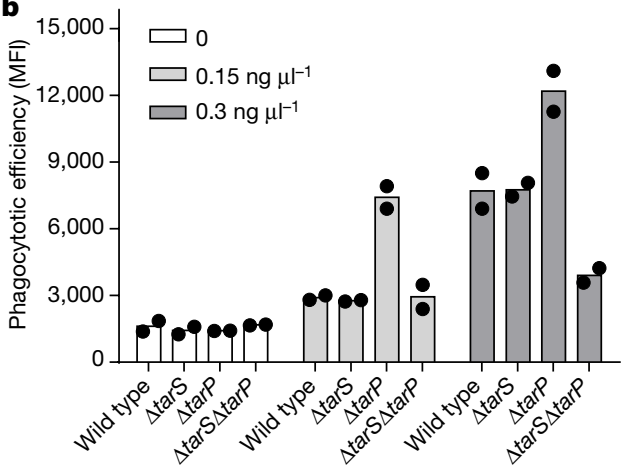

Fig. 4 TarP attenuates immunogenicity of WTA. a, TarP expression reduces deposition of IgG from human serum on N315 cells. The protein A gene spa was deleted in all strains. Top, human IgG isolated from three individual healthy donors (A, B, and C; $n=4)$; bottom, left, IgG from human serum enriched for RN4220 WTA binding $(n=4)$; middle and right, pooled human IgG from different suppliers (Abcam, $n=4$; Athens $\mathrm{R} \& \mathrm{~T}, n=6)$. Results were normalized against wild type and shown as means with s.d. of $n$ experiments. $P$ values for comparison with wild type were calculated by one-way ANOVA with Dunnett's post-test (two-sided), and $P \leq 0.05$ was considered significant. Significant $P$ values are displayed. b, TarP reduces neutrophil phagocytosis of $\mathrm{N} 315$ strains lacking protein A, opsonized with indicated concentrations of IgG enriched for WTA binding. Values are depicted as mean fluorescence intensity (MFI). Means of two dependent replicates of a representative experiment are shown.

preparations for their capacity to opsonize a panel of N315 strains with or without $\operatorname{tar} P$ and/or tarS. The mutant lacking any WTA glycosylation bound the lowest amount of IgG compared to WTA glycosylation-positive strains (Fig. 4a), demonstrating that glycosylated WTA is a prominent $S$. aureus antigen in humans. Exclusive expression of tarS led to strongly increased IgG binding compared to the glycosylation-deficient mutant, indicating that $\beta$-GlcNAc on RboP C4 is an important epitope for human anti-S. aureus antibodies. By contrast, expression of tarP in the presence or absence of tarS led to only slightly increased IgG binding compared to the glycosylation-deficient mutant. The capacity of TarP to impair the deposition of IgG on S. aureus differed with individual serum donors and reached average levels in pooled serum preparations (Fig. 4a). When $\operatorname{tar} P$ was deleted in three further CC5 isolates, they showed similarly increased capacities to bind human serum antibodies compared to the wild-type strains (Extended Data Fig. 1e). Additionally, tarP deletion led to a substantially increased capacity of human neutrophils to phagocytose opsonized S. aureus (Fig. 4b, Extended Data Fig. 1g). Thus, only a small percentage of $S$. aureus-specific antibodies can bind WTA with $\beta$-GlcNAc on RboP C3, and tarPexpressing $S$. aureus are less likely to be detected and eliminated by human phagocytes. TarP-modified WTA.

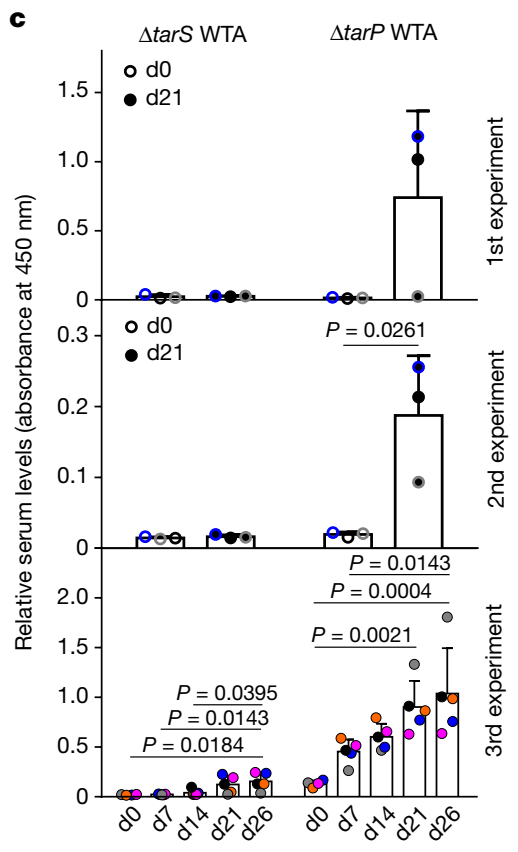

The other two representative experiments can be found in Extended Data Fig. 1g. c, TarP abrogates IgG response of mice towards WTA. For each experiment, WTA from N315 $\Delta \operatorname{tar} P$ or $\Delta \operatorname{tar} S$ was isolated independently. At least three mice per group were vaccinated and analysed for specific $\mathrm{IgG}$ at indicated time points after vaccination. Results are depicted as mean absorbance with s.d. Individual mice are indicated by colour. Increase in IgG levels was assessed by one-way ANOVA with Tukey's post-test (two-sided). Significant differences $(P \leq 0.05)$ are indicated with corresponding $P$ values. d, Vaccination with WTA does not protect mice against $\operatorname{tar} P$-expressing N315, as shown for bacterial loads in kidney upon intravenous infection. No significant differences between groups of either five vaccinated mice or four mice for the alum control group (means indicated), calculated by one-way ANOVA, were observed.

We purified N315 WTA that had been glycosylated by TarS or TarP and used it to immunize mice. Antibodies binding to regular (TarS-modified) WTA increased continuously over three weeks after vaccination (Fig. 4c). By contrast, no or only very low amounts of IgG directed against TarP-glycosylated WTA emerged, indicating that WTA modified at RboP C3 is much less immunogenic than WTA modified at RboP C4. This experiment was repeated three times with three different WTA preparations and yielded broadly similar data.

Vaccination with $S$. aureus WTA bearing GlcNAc at RboP C4 protects mice against infection by CA-MRSA strains USA300 (CC8) or USA400 (CC1), which both lack $\operatorname{tar} P^{5,21}$. Remarkably, vaccination with regular (TarS-modified) or TarP-modified WTA did not lead to any notable protection against subsequent infection with tarP-expressing N315 compared to mock vaccination, despite the robust antibody response against regular WTA (Fig. 4d). Together, our results demonstrate that tarP protects $S$. aureus against adaptive host defences by allowing bacteria to evade recognition by preexisting antiS. aureus antibodies and by exploiting the poor immunogenicity of

It is possible that TarP-modified WTA mimics a currently unknown autoantigen and is therefore hardly immunogenic. On the other hand, regular S. aureus WTA can be ingested by antigen-presenting cells 
and presented to T cells, in a largely unexplored way, thereby evoking specific immunoglobulins and immunological memory ${ }^{22,23}$. It is possible that TarP-modified WTA is refractory to this process. Thus, TarSand TarP-modified WTA could be helpful for decoding glycopolymer presentation pathways and for defining the most promising WTA epitopes for the development of protective vaccines against $S$. aureus.

Protection against $S$. aureus infections is urgently needed, in particular for hospitalized and immunocompromised patients $s^{2,4}$. Antibodies can in principle protect against $S$. aureus, but their titres and specificities vary largely among humans and they are often not protective in immunocompromised patients ${ }^{3}$, probably in particular against S. aureus clones that mask dominant epitopes, for instance using TarP. Unfortunately, all previous human vaccination attempts with protein or glycopolymer antigens have failed, for reasons that are unclear ${ }^{24}$. Our study identifies a new strategy used by pandemic MRSA clones to subvert antibody-mediated immunity, which should be considered in future vaccination approaches. S. aureus WTA with GlcNAc at RboP C3 has been reported as a type-336 antigen, but was not further explored ${ }^{25}$. We found that $\operatorname{tar} P$ is present in type-336 S. aureus (Extended Data Fig. 1f). However, TarP-modified WTA is a very poor antigen and vaccines directed against GlcNAc at WTA RboP C3 or C4 may fail against many of the pandemic MRSA clones. The structural characterization of TarP will instruct the development of specific TarP inhibitors that could become important in combination with anti-WTA vaccines or antibiotic therapies. We found tarP-encoding prophages in $70-80 \%$ of south-west German HA-MRSA CC5 and $40 \%$ of Danish LA-MRSA CC398 isolates (Extended Data Table 1), pointing to a crucial role of $\operatorname{tar} P$ in the fitness of these lineages and raising concerns of further dissemination by horizontal gene transfer. TarP is a new and probably crucial component of the $S$. aureus virulence factor arsenal ${ }^{26,27}$, highlighting the important roles of adaptive immunity and its evasion in S. aureus infections.

\section{Online content}

Any methods, additional references, Nature Research reporting summaries, source data, statements of data availability and associated accession codes are available at https://doi.org/10.1038/s41586-018-0730-x.

Received: 28 June 2018; Accepted: 18 October 2018; Published online 21 November 2018.

1. Tong, S. Y., Davis, J. S., Eichenberger, E., Holland, T. L. \& Fowler, V. G. Jr. Staphylococcus aureus infections: epidemiology, pathophysiology, clinical manifestations, and management. Clin. Microbiol. Rev. 28,603-661 (2015).

2. Lee, A. S. et al. Methicillin-resistant Staphylococcus aureus. Nat. Rev. Dis. Primers 4, 18033 (2018)

3. Stentzel, S. et al. Specific serum IgG at diagnosis of Staphylococcus aureus bloodstream invasion is correlated with disease progression. J. Proteomics 128 1-7 (2015).

4. Missiakas, D. \& Schneewind, O. Staphylococcus aureus vaccines: deviating from the carol. J. Exp. Med. 213, 1645-1653 (2016).

5. Lehar, S. M. et al. Novel antibody-antibiotic conjugate eliminates intracellular S. aureus. Nature 527, 323-328 (2015)

6. Weidenmaier, C. \& Peschel, A. Teichoic acids and related cell-wall glycopolymers in Gram-positive physiology and host interactions. Nat. Rev. Microbiol. 6, 276-287 (2008).

7. Brown, S. et al. Methicillin resistance in Staphylococcus aureus requires glycosylated wall teichoic acids. Proc. Natl Acad. Sci. USA 109, 18909-18914 (2012).

8. Tacconelli, E et al. Discovery, research, and development of new antibiotics: the WHO priority list of antibiotic-resistant bacteria and tuberculosis. Lancet Infect. Dis. 18, 318-327 (2018)

9. Kurokawa, K. et al. Glycoepitopes of staphylococcal wall teichoic acid govern complement-mediated opsonophagocytosis via human serum antibody and mannose-binding lectin. J. Biol. Chem. 288, 30956-30968 (2013).

10. Lee, J. H. et al. Surface glycopolymers are crucial for in vitro anti-wall teichoic acid IgG-mediated complement activation and opsonophagocytosis of Staphylococcus aureus. Infect. Immun. 83, 4247-4255 (2015).

11. Winstel, V. et al. Wall teichoic acid structure governs horizontal gene transfer between major bacterial pathogens. Nat. Commun. 4, 2345 (2013).

12. Nübel, U. et al. Frequent emergence and limited geographic dispersal of methicillin-resistant Staphylococcus aureus. Proc. Natl Acad. Sci. USA 105, 14130-14135 (2008)

13. McCarthy, A. J. \& Lindsay, J. A. Staphylococcus aureus innate immune evasion is lineage-specific: a bioinfomatics study. Infect. Genet. Evol. 19, 7-14 (2013).
14. Bal, A. M. et al. Genomic insights into the emergence and spread of international clones of healthcare-, community- and livestock-associated meticillin-resistant Staphylococcus aureus: Blurring of the traditional definitions. J. Glob. Antimicrob. Resist. 6, 95-101 (2016).

15. Hau, S. J., Bayles, D. O., Alt, D. P., Frana, T. S. \& Nicholson, T. L. Draft genome sequences of 63 swine-associated methicillin-resistant Staphylococcus aureus sequence type 5 isolates from the United States. Genome Announc. 5 , e01081-17 (2017)

16. Xia, G. et al. Wall teichoic acid-dependent adsorption of staphylococcal siphovirus and myovirus. J. Bacteriol. 193, 4006-4009 (2011).

17. Vinogradov, E., Sadovskaya, I., Li, J. \& Jabbouri, S. Structural elucidation of the extracellular and cell-wall teichoic acids of Staphylococcus aureus MN8m, a biofilm forming strain. Carbohydr. Res. 341, 738-743 (2006).

18. Sobhanifar, S. et al. Structure and mechanism of Staphylococcus aureus TarS, the wall teichoic acid $\beta$-glycosyltransferase involved in methicillin resistance. PLoS Pathog. 12, e1006067 (2016)

19. Lairson, L. L., Henrissat, B., Davies, G. J. \& Withers, S. G. Glycosyltransferases: structures, functions, and mechanisms. Annu. Rev. Biochem. 77, 521-555 (2008).

20. Kozmon, S. \& Tvaroska, I. Catalytic mechanism of glycosyltransferases: hybrid quantum mechanical/molecular mechanical study of the inverting $\mathrm{N}$-acetylglucosaminyltransferase I. J. Am. Chem. Soc. 128, 16921-16927 (2006).

21. Takahashi, K. et al. Intradermal immunization with wall teichoic acid (WTA) elicits and augments an anti-WTA IgG response that protects mice from methicillin-resistant Staphylococcus aureus infection independent of mannose-binding lectin status. PLoS One 8, e69739 (2013).

22. Weidenmaier, C., McLoughlin, R. M. \& Lee, J. C. The zwitterionic cell wall teichoic acid of Staphylococcus aureus provokes skin abscesses in mice by a novel CD4+ T-cell-dependent mechanism. PLoS One 5, e13227 (2010).

23. Wanner, S. et al. Wall teichoic acids mediate increased virulence in Staphylococcus aureus. Nat. Microbiol. 2, 16257 (2017).

24. Pozzi, C. et al. Vaccines for Staphylococcus aureus and target populations. Curr. Top. Microbiol. Immunol. 409, 491-528 (2017)

25. Fattom, A., Sarwar, J., Kossaczka, Z., Taylor, K. \& Ennifar, S. Method of protecting against staphylococcal infection. US Patent US20060228368A1 (2006).

26. Thammavongsa, V., Kim, H. K., Missiakas, D. \& Schneewind, O. Staphylococcal manipulation of host immune responses. Nat. Rev. Microbiol. 13, 529-543 (2015).

27. Spaan, A. N., Surewaard, B. G., Nijland, R. \& van Strijp, J. A. Neutrophils versus Staphylococcus aureus: a biological tug of war. Annu. Rev. Microbiol. 67, 629-650 (2013).

Acknowledgements We thank S. Popovich and P. Kühner for technical assistance; E. Weiß for help with phagocytosis experiments; R. Rosenstein and $X$. Li for discussions; B. Blaum and G. Zocher for assistance with NMR analysis and support for structure phasing and discussion; and the Swiss Lightsource beamline staff of the Paul Scherrer Institute for beam time and technical support. This work was financed by grants from the German Research Foundation to A.P. (TRR34, CRC766, TRR156, RTG1708), T.S. (TRR34, CRC766), C.W. (TRR34, CRC766, TRR156, RTG1708), and G.X. (CRC766); the German Center of Infection Research to A.P. (HAARBI); the Ministry of Science and Technology, Thailand Government to W.S.; the Korean Drug Development Foundation to S.-H.K. and B.L.L. (KDDF-201703-1); and the Max-PlanckSociety to P.H.S.

Reviewer information Nature thanks M. Crispin, F. DeLeo, M. Gilmore and J. Zimmer for their contribution to the peer review of this work.

Author contributions D.G. characterized TarP in vivo and its genomic context, created mutants, designed experiments, purified WTA, and performed experiments with human IgGs. Y.G. designed experiments, purified proteins, crystallized proteins, solved the structures, and performed in vitro analysis of TarP. C.D.C. performed NMR experiments. C.D.C. and A.M. analysed the NMR data and wrote the NMR discussion. S.-H.K. performed and B.L.L. designed and interpreted mouse immunization and infection experiments. K.S. designed IgG deposition experiments. B.S. and C.W. collected and characterized CC5 MRSA strains. J.L collected and characterized CC398 strains. J.L and C.W. analysed S. aureus genomes. F.-F.X, C.P., and P.H.S. designed and synthesized 3RboP. S.A. and J.C. designed and synthesized 6 RboP- $\left(\mathrm{CH}_{2}\right)_{6} \mathrm{NH}_{2}$. W.S. performed MIC experiments. G.X. identified $\operatorname{tar} P$, and characterized and interpreted MIC data D.G., Y.G., A.P., T.S., and G.X. designed the study, analysed results, and wrote the paper.

\section{Competing interests The authors declare no competing interests.}

\section{Additional information}

Extended data is available for this paper at https://doi.org/10.1038/s41586018-0730-x.

Supplementary information is available for this paper at https://doi.org/ 10.1038/s41586-018-0730-x.

Reprints and permissions information is available at http://www.nature.com/ reprints.

Correspondence and requests for materials should be addressed to T.S. or A.P. Publisher's note: Springer Nature remains neutral with regard to jurisdictional claims in published maps and institutional affiliations. 


\section{METHODS}

No statistical methods were used to predetermine sample size. The experiments were not randomized. The investigators were not blinded to allocation during experiments and outcome assessment.

Bacterial strains and growth conditions. S. aureus strains N315, RN4220, and MW2 (wild type and mutants) were used for this study. Collections of CC5 isolates of the Rhine-Hesse pulsed-field gel electrophoresis type ${ }^{28}$ and of the LA-MRSA lineage CC398 from the Danish Statens Serum Institut ${ }^{29,30}$ were analysed for the presence of $\operatorname{tar} P$ and for podophage susceptibility. Additionally, 48 spa-type t002 (ST5) and 16 spa-type t003 (ST225) isolates were obtained from the MRSA collection of the University Hospital Tübingen and analysed for tarP presence by PCR. S. aureus strains were cultivated in tryptic soy broth (TSB) or basic medium (BM; $1 \%$ tryptone, $0.5 \%$ yeast extract, $0.5 \% \mathrm{NaCl}, 0.1 \%$ glucose, $0.1 \% \mathrm{~K}_{2} \mathrm{HPO}_{4}, \mathrm{w} / \mathrm{v}$ ). MICs of oxacillin were determined by microbroth dilution according to established guidelines ${ }^{31}$

Experiments with phages. $\operatorname{tar} P$-encoding phages were identified in genome sequences using the webtool Phaster ${ }^{32}$ in representative strains listed with GenBank accession: $\Phi$ tarP-Sa3int with immune evasion cluster (IEC) in CC5 (strain N315, BA000018.3), $\operatorname{tar} P$-Salint, found in LA-MRSA of CC5 (strain ISU935, CP017090), and tarP-Sa9int found in CC398 (strain E154, CP013218)

Phage susceptibility was determined using a soft-agar overlay method ${ }^{16}$. In brief, $10 \mu \mathrm{l}$ phage lysate of $10^{4}-10^{6} \mathrm{PFU}$ was dropped onto soft agar containing 100 $\mu \mathrm{l}$ bacterial suspension $\left(\mathrm{OD}_{600}\right.$ of 0.1$)$. Plates were incubated at $37^{\circ} \mathrm{C}$ overnight. The efficiency of plating was determined as described ${ }^{33}$. Transfer of SaPIs was determined according to previously described methods ${ }^{11}$. In brief, SaPI particle lysates were generated from S. aureus strain JP1794, which encodes a SaPI with a resistance marker for tetracycline ${ }^{34}$. PFU of SaPI lysate was determined on RN4220. $200 \mu \mathrm{l}$ bacterial culture $\left(\mathrm{OD}_{600}\right.$ of 0.5$)$ was mixed with $100 \mu \mathrm{l}$ of SaPI particle lysate (SaPIbovl $\left.(\Phi 11), 10^{6} \mathrm{PFU} / \mathrm{ml}\right)$, incubated at $37^{\circ} \mathrm{C}$ for $15 \mathrm{~min}$. Appropriate dilutions were plated on TSB plates containing $3 \mu \mathrm{g} / \mathrm{ml}$ of tetracycline, and CFU were checked after overnight incubation.

WTA isolation and structure analysis. WTA from S. aureus was isolated and purified according to previously described methods ${ }^{11}$. In brief, WTA was released from purified peptidoglycan by treatment with $5 \%$ trichloroacetic acid and dialysed extensively against water using a Spectra/Por3 dialysis membrane (MWCO of $3.5 \mathrm{kDa}$; VWR International $\mathrm{GmbH}$ ). Obtained soluble WTA was quantified by determining the content of phosphate ${ }^{35}$ and $\mathrm{GlcNAc}^{36}$. For PAGE analysis of WTA, samples ( $400 \mathrm{nmol}$ of phosphate per lane) were applied to a $26 \%$ polyacrylamide (Rotiphorese Gel 40 (19:1)) resolving gel and separated at $25 \mathrm{~mA}$ for $16 \mathrm{~h}^{37}$. The gel was equilibrated in a solution of $40 \%$ ethanol and $5 \%$ acidic acid at room temperature for $1 \mathrm{~h}$ and the WTA ladders were visualized by incubation with alcian blue $(0.005 \%)$ for several hours.

NMR spectroscopy experiments were carried out on a Bruker DRX-600 spectrometer equipped with a cryo-probe, at $288 \mathrm{~K}$ (WT-WTA, TarS-WTA, and TarP-WTA) or $298 \mathrm{~K}$ (double-mutant WTA lacking any glycosylation). Chemical shifts of spectra recorded in $\mathrm{D}_{2} \mathrm{O}$ were calculated in p.p.m. relative to internal acetone (2.225 and 31.45 p.p.m.). The spectral width was set to 10 p.p.m. and the frequency carrier placed at the residual HOD peak, suppressed by pre-saturation. Two-dimensional spectra (TOCSY, gHSQC, gHMBC, and HSQC-TOCSY) were measured using standard Bruker software. For all experiments, 512 FIDs of 2,048 complex data points were collected, 32 scans per FID were acquired for homonuclear spectra, and 20 or $100 \mathrm{~ms}$ of mixing time was used for TOCSY spectra. Heteronuclear ${ }^{1} \mathrm{H}_{-}{ }^{13} \mathrm{C}$ spectra were measured in the ${ }^{1} \mathrm{H}$-detected mode, gHSQC spectrum was acquired with 40 scans per FID, the GARP sequence was used for ${ }^{13} \mathrm{C}$ decoupling during acquisition; gHMBC scans doubled those of gHSQC spectrum. As for HSQC-TOCSY, the multiplicity editing during selection step version was used, scans tripled those of the HSQC spectrum and two experiments were acquired by setting the mixing time to 20 or $80 \mathrm{~ms}$. During processing, each data matrix was zero-filled in both dimensions to give a matrix of $4 \mathrm{~K} \times 2 \mathrm{~K}$ points and was resolution-enhanced in both dimensions by a cosine-bell function before Fourier transformation; data processing and analysis were performed with the Bruker Topspin 3 program.

Molecular biology. All primers used for PCR, cloning, and mutagenesis are listed in Supplementary Table 1. $\operatorname{tar} P$ (UniProt A0A0H3JNB0, NCBI Gene ID 1260584) was amplified using genomic DNA of S. aureus N315 and inserted in Eschericha coli/S. aureus shuttle vector pRB $474^{38}$ at the BamHI and SacI sites, to transform S. aureus, or into $\mathrm{pQE} 80 \mathrm{~L}$ at BamH1 and HindIII sites, to transform E. coli BL21(DE3). A thrombin cleavage site was inserted between the His-tag and mature protein in $\mathrm{PQE} 80 \mathrm{~L}$. Single mutations of TarP were introduced by PCRbased site-directed mutagenesis ${ }^{39}$. The obtained amplicons were confirmed by sequencing. For the construction of marker-less $S$. aureus deletion mutants of tarS or $\operatorname{tar} P$, the pIMAY shuttle vector was used ${ }^{40}$. The IgG-binding surface protein A gene $(s p a)$ was deleted using the pKORI shuttle vector ${ }^{41}$. Protein A deletion had no impact on phage siphophage or podophage susceptibility, indicating that it did not alter WTA amount or structure.

Protein expression, purification, and activity assay. E. coli BL21(DE3) were grown in $\mathrm{LB}$ medium at $30^{\circ} \mathrm{C}$. Expression of $\operatorname{tar} P$ was induced with $1 \mathrm{mM} \mathrm{IPTG}$ at $22^{\circ} \mathrm{C}$ at an $\mathrm{OD}_{600}$ of 0.6 . After $15 \mathrm{~h}$, cells were harvested, washed with wash buffer $(50 \mathrm{mM}$ Tris- $\mathrm{HCl}, \mathrm{pH}$ 8.0, $1 \mathrm{mM}$ EDTA), and lysed by sonication with lysis buffer (70 $\mathrm{mM}$ $\mathrm{NaH}_{2} \mathrm{PO}_{4}, \mathrm{pH} 8.0,1 \mathrm{M} \mathrm{NaCl}, 20 \%$ glycerol, $10 \mathrm{U} / \mathrm{ml}$ of benzonase nuclease). After centrifugation $(15,000 \mathrm{~g})$, the supernatant was filtered with a $0.45 \mu \mathrm{m}$ filter, loaded onto a His Trap FF column (GE Healthcare, $5 \mathrm{ml}$ ), and washed with buffer A ( $50 \mathrm{mM} \mathrm{NaH}_{2} \mathrm{PO}_{4}, \mathrm{pH} 8.0,1 \mathrm{M} \mathrm{NaCl}, 20 \%$ glycerol) supplemented with $45 \mathrm{mM}$ imidazole and buffer B (buffer A with $90 \mathrm{mM}$ imidazole). Finally, the protein was eluted with buffer C (buffer A with $500 \mathrm{mM}$ imidazole), and the fractions were pooled, and further purified by size-exclusion chromatography on a Superdex 200 10/30 column equilibrated with buffer D (20 mM MOPS, pH 7.6, $400 \mathrm{mM}$ $\mathrm{LiCl}, 10 \mathrm{mM} \mathrm{MgCl}_{2}, 5 \mathrm{mM} \beta$-mercaptoethanol, $5 \%$ glycerol). The peak fractions were pooled and concentrated to $1.4 \mathrm{mg} / \mathrm{ml}$ for crystallization. For selenomethionyl-form TarP production, bacteria were grown in a selenomethionine-containing medium (Molecular Dimension) and auto-induction was carried out. The protein was purified as described above. The activity of wild-type and mutated TarP, as well as donor substrate specificity of TarP were determined with the ADP Quest Assay kit (DiscoverRx, Extended Data Tables 2, 3). The reaction volume was $20 \mu \mathrm{l}$ with $1 \mathrm{mM}$ UDP-GlcNAc, $1.5 \mathrm{mM}$ purified WTA from RN4220 $\Delta$ tarM/S. The reaction was started with protein and incubated at room temperature for $1 \mathrm{~h}$. Released UDP, coupled into a fluorescence signal, was detected in a 384-well black assay plate with $530 \mathrm{~nm}$ excitation and $590 \mathrm{~nm}$ emission wavelengths using TECAN Infinite M200. Crystallization and data collection. Crystals were obtained by vapour diffusion at $20^{\circ} \mathrm{C} .1 \mu \mathrm{l}$ protein solution was mixed with $1 \mu \mathrm{l}$ reservoir solution containing $25 \%$ PEG 3350, $250 \mathrm{mM} \mathrm{MgCl}_{2}$, and $0.1 \mathrm{M}$ sodium citrate, $\mathrm{pH}$ 5.7. The selenomethionyl-form protein was crystallized under the same conditions. For crystals of TarP with UDP-GlcNAc, $27 \mathrm{mM}$ UDP-GlcNAc was introduced in the reservoir solution containing $250 \mathrm{mM} \mathrm{MgCl}_{2}$ or $230 \mathrm{mM} \mathrm{MnCl}_{2}$. Crystals of TarP with $\mathrm{Mg}^{2+}$ were used for soaking of synthetic $3 \mathrm{RboP}(60 \mathrm{mM}), 6 \mathrm{RboP}-\left(\mathrm{CH}_{2}\right)_{6} \mathrm{NH}_{2}(41 \mathrm{mM})$, or UDP-GlcNAc $(20 \mathrm{mM})$ combined with $3 \mathrm{RboP}(52 \mathrm{mM})$ for $5 \mathrm{~min}$. For data collection the crystals were cryo-protected with $20 \%$ glycerol in reservoir solution and flash-frozen in liquid nitrogen. Diffraction data were collected at beamline X06DA of Swiss Light Source in Villigen, Switzerland, or at beamline BL14.1 at BESSY-II, Helmholtz Zentrum Berlin.

Phasing, model building, and refinement. For phase determination, two data sets from a selenomethionine-containing TarP crystal were collected at wavelengths of $0.97941 \AA$ (peak) and $0.97952 \AA$ (inflection). The structure was solved by multiwavelength anomalous dispersion (MAD) at $2.60 \AA$ resolution. All data were reduced using XDS/XSCALE software packages ${ }^{42}$. Initial phases were derived from the substructure of 26 selenium atom sites per asymmetric unit with the program suite SHELX C/D/E $/ \mathrm{E}^{43}$. The heavy atom parameters were further refined and the initial phases were improved by SHARP/autoSHARP ${ }^{44}$. The initial model was generated with PHENIX ${ }^{45}$ and the final model was achieved by cycles of iterative model modification using $\mathrm{COOT}^{46}$, and restrained refinement with REFMAC. TLS was used in the later stages ${ }^{47,48}$. The four binary and one ternary complex structures were solved by molecular replacement using $\mathrm{PHASER}^{49}$ and the unliganded TarP structure was used as a search model. UDP-GlcNAc, $3 \mathrm{RboP}, \mathrm{Mg}^{2+}$, or $\mathrm{Mn}^{2+}$ were removed from the models to calculate the simulated annealing $\left(\mathrm{m} F_{\mathrm{o}}-\mathrm{D} F_{\mathrm{c}}\right)$ omit maps using PHENIX. The anomalous difference map of $\mathrm{Mn}^{2+}$ at $1.89259 \AA$. was generated by FFT within CCP4, from which two $\mathrm{Mn}^{2+}$ in the active site and one $\mathrm{Mn}^{2+}$ at the trimer interface were identified. The coordinate and parameter files for $3 \mathrm{RboP}$ and $6 \mathrm{RboP}-\left(\mathrm{CH}_{2}\right)_{6} \mathrm{NH}_{2}$ were calculated using the PRODRG server ${ }^{50}$. The structure figures were generated by $\mathrm{PyMOL}^{51}$ and the models were evaluated using MolProbity ${ }^{52}$. Statistics for the data collection, phasing, and refinement are reported in Extended Data Tables 4 and 5.

Synthesis of ribitol phosphate oligomers. Synthesis of $3 R b o P$. Target compound 1, D-ribitol-5-phosphate trimer (3RboP), was prepared by the phosphoramidite method $^{53,54}$ (Supplementary Fig. 2). In brief, the primary alcohol of commercially available compound 2 was converted into levulinoyl ester by using levulinic acid and $N, N^{\prime}$-dicyclohexylcarbodiimide (DCC), and the allyl group of $\mathbf{3}$ was removed with tetrakis(triphenylphosphine)palladium to produce compound 4 . The primary alcohol of $\mathbf{4}$ reacted with phosphine derivative $\mathbf{5}$ in the presence of diisopropylammonium tetrazolide ${ }^{55}$ to generate phosphoramidite 6 . At the same time, compound 4 was coupled with dibenzyl $N, N$-diisopropylphosphoramidite 7 , which was catalysed by $1 H$-tetrazole, and the product was further oxidized by tert-butyl hydroperoxide, yielding protected D-ribitol-5-phosphate 8. Cleavage of the levulinoyl ester of $\mathbf{8}$ with hydrazine hydrate resulted in benzyl protected D-ribitol-5-phosphate $\mathbf{9}$, which was further coupled with phosphoramidite 6 and oxidized with tert-butyl hydroperoxide to yield protected dimers of D-ribitol-5-phosphate 10. After removal of the levulinoyl group, the dimer $\mathbf{1 1}$ was coupled with phosphoramidite $\mathbf{6}$ using the same conditions as above to obtain a protected trimer of D-ribitol-5-phosphate 
12. Subsequent removal of the levulinoyl group and hydrogenolysis of $\mathbf{1 3}$ to remove all benzyl groups yielded 3RboP 1 . All chemicals and experimental procedures as well as characterization of products can be found in the Supplementary Methods. Synthesis of $6 \mathrm{RboP}-\left(\mathrm{CH}_{2}\right)_{6} \mathrm{NH}_{2}$. Aminohexyl D-ribitol-5-phosphate hexamer (6RboP- $\left.\left(\mathrm{CH}_{2}\right)_{6} \mathrm{NH}_{2}\right)$ was synthesized using a new method (Supplementary Fig. 3). All chemicals (Acros, Biosolve, Sigma-Aldrich and TCI) for the synthesis were used as received and all reactions were performed under a protective argon atmosphere at room temperature, unless otherwise stated. Procedures for phosphoramidite coupling, oxidation, detritylation, global deprotection, TLC analysis and characterization of these compounds can be found in Supplementary Methods.

Human samples. Venous blood samples were obtained from male and female healthy volunteers (20-50 years) with protocols approved by the Institutional Review Board for Human Subjects at the University of Tübingen (014/2014BO2 und 549/2018BO2). Informed written consent was obtained from all volunteers. Blood samples were used for purification of either serum IgGs or neutrophils as described below.

IgG from human plasma. IgG was purified from plasma of human donors using the NAb Protein G Spin Kit (ThermoFisher), purity was checked by SDS PAGE, and protein concentration was determined using Bradford assay. Anti-WTA-IgG was prepared as described ${ }^{9}$. To analyse the IgG-binding capacity of $S$. aureus cells, exponentially growing bacterial cultures were adjusted to an $\mathrm{OD}_{600}$ of 0.5 , diluted $1: 10$ in PBS, and $100 \mu \mathrm{l}$ of diluted bacteria was mixed with $100 \mu \mathrm{l}$ of IgG diluted in PBS with $1 \%$ BSA. The concentration of IgG was $250 \mathrm{ng} / \mathrm{ml}$ for IgG enriched for WTA binding, $10 \mu \mathrm{g} / \mathrm{ml}$ for IgG from pooled human serum (Athens R\&T 16-16-090707, Abcam ab98981), or $5 \mu \mathrm{g} / \mathrm{ml}$ for single-donor IgG preparations. A control without IgG was included in all experiments for all mutants. Samples were incubated at $4^{\circ} \mathrm{C}$ for $1 \mathrm{~h}$, centrifuged, washed 2-3 times with PBS, and further incubated with $100 \mu$ l FITC-labelled anti-human IgG (Thermo Scientific, 62-8411, 1:100 in PBS with $1 \% \mathrm{BSA}, 62-8411$ ) at $4{ }^{\circ} \mathrm{C}$ for $1 \mathrm{~h}$. Bacteria were centrifuged, washed 2-3 times with PBS, and fixed with $2 \%$ paraformaldehyde (PFA). Surface-bound IgG was quantified by flow cytometry using a BD FACSCalibur. For all flow cytometry experiments a mutant panel lacking spa, the gene for the IgG-binding protein $\mathrm{A}$, was used. The subsequent gating strategy is exemplified in Extended Data Fig. $5 \mathrm{a}$. IgG-mediated phagocytosis. Stationary-phase S. aureus cells were washed once with PBS and labelled by incubation in PBS containing $10 \mu \mathrm{M}$ carboxyfluorescein succinimidyl ester (CFSE; $\mathrm{OD}_{600}$ of 1.7 ) at $37^{\circ} \mathrm{C}$ for $1 \mathrm{~h}$. The bacteria were washed three times and resuspended in PBS. CFU were determined by plating on TSB plates and bacteria were heat-inactivated at $70{ }^{\circ} \mathrm{C}$ for $20 \mathrm{~min}$. CFSE-labelled S. aureus $\left(1 \times 10^{7} \mathrm{cells} / \mathrm{ml}\right)$ in PBS with $0.5 \%$ BSA were opsonized with anti-WTAIgG $(0.15$ or $0.3 \mathrm{ng} / \mu \mathrm{l})$ at $4^{\circ} \mathrm{C}$ for $40 \mathrm{~min}$. Neutrophils from human donors, isolated via Ficoll-Histopaque density gradient centrifugation ${ }^{56}$, were diluted to a concentration of $2.5 \times 10^{6} / \mathrm{ml}$ in neutrophil medium ( $10 \% \mathrm{HSA}, 2 \mathrm{mM}$ L-glutamine, $2 \mathrm{mM}$ sodium pyruvate, $10 \mathrm{mM}$ HEPES). $200 \mu \mathrm{l}$ neutrophil suspension was incubated with $25 \mu \mathrm{l}$ opsonized bacteria (final MOI 0.5) in a 96-well plate at $37^{\circ} \mathrm{C}$ for $30 \mathrm{~min}$, centrifuged ( $350 \mathrm{~g}, 10 \mathrm{~min}$ ), washed once with $200 \mu \mathrm{l}$ PBS, and fixed with $2 \%$ PFA at room temperature for $15 \mathrm{~min}$. Cells were washed twice with PBS and analysed by flow cytometry, whereby surface-bound and ingested bacteria were measured without discrimination. An example of the neutrophil gating strategy can be found in Extended Data Fig. 5 b.

Mice. Six-week-old sex-matched wild-type C57BL/6J mice, purchased from ORIENT BIO (Charles River Breeding Laboratories in Korea), were kept in micro-isolator cages in a pathogen-free animal facility. The conducted experiments were performed according to guidelines and approval (PNU-2017-1503) by the Pusan National University-Institutional Animal Care and Use Committee (PNU-IACUC). Sample size was chosen to obtain significant outcomes (alpha error $\leq 5 \%$ ), based on results from previous experiments ${ }^{21}$. Experiments were performed in a non-blinded, non-randomized fashion.

Mouse vaccination and infection. $30 \mu \mathrm{g}$ of purified WTA from S. aureus N315 wild-type or isogenic $\Delta \operatorname{tar} P$, or $\Delta \operatorname{tar} S$ mutants was dissolved in $15 \mu \mathrm{l} \mathrm{PBS}$ and mixed with the same volume of aluminium hydroxide gel adjuvant (Alhydrogelr $1.3 \%, 6.5 \mathrm{mg} / \mathrm{ml}$, Brennatag). The mixtures were incubated at $37^{\circ} \mathrm{C}$ with agitation for $1 \mathrm{~h}$ and injected three times at one-week intervals via mouse footpads. Seven days after the third injection, blood was obtained from the retro-orbital sinus and centrifuged $(9,000 \mathrm{~g})$ at $4^{\circ} \mathrm{C}$ for $10 \mathrm{~min}$. The supernatants were aliquoted $(50 \mu \mathrm{l})$ and stored at $-80^{\circ} \mathrm{C}$ for ELISA quantification of WTA-binding IgG as described ${ }^{57}$. Sera were diluted 1:100 and tested by ELISA on 96-well plates coated with $2.5 \mu \mathrm{g} / \mathrm{ml}$ sonicated WTA preparations (WTA from N315, $\Delta \operatorname{tarS}$ or $\Delta \operatorname{tar} P$, respectively).

To prepare an inoculum for infection, N315 wild-type bacteria were grown in TBS at $37^{\circ} \mathrm{C}$ with agitation ( 180 r.p.m.) until they reached an $\mathrm{OD}_{600}$ of 1.0. After centrifugation $(3,500 \mathrm{~g})$ at $4^{\circ} \mathrm{C}$ for $10 \mathrm{~min}$, bacteria adjusted to $5 \times 10^{7} \mathrm{CFU}$ in $50 \mu \mathrm{l}$ PBS containing $0.01 \%$ BSA were intravenously injected ( $n=5$ per group). Injected bacterial numbers were verified by plating serial dilutions of the inoculum onto TSA plates. To determine residual bacterial dissemination to kidneys, challenged mice were euthanized, and organs were extracted aseptically and homogenized in $1 \mathrm{ml}$ of saline using a Polytron homogenizer (PT3100). The homogenates were serially diluted and plated on TSA to determine CFU counts. CFU were calculated per $1 \mathrm{ml}$ of kidney.

Statistical analyses. Statistical analysis was performed by using GraphPad Prism (GraphPad Software, Inc.). Statistically significant differences were calculated by appropriate statistical methods as indicated. $P$ values of $\leq 0.05$ were considered significant.

Reporting summary. Further information on experimental design is available in the Nature Research Reporting Summary linked to this paper.

\section{Data availability}

All major data generated or analysed in this study are included in the article or its supplementary information files. The coordinates and structure factors were deposited in the Protein Data Bank under accession numbers 6H1J, 6H21, 6H2N, $6 \mathrm{H} 4 \mathrm{~F}, 6 \mathrm{H} 4 \mathrm{M}$ and 6HNQ. Source data for experiments with animals (Fig. 4c, d) are provided. Additionally, a gel image of Extended Data Fig. If is supplied as Supplementary Fig. 1. All other data relating to this study are available from the corresponding authors on reasonable request.

28. Schulte, B., Bierbaum, G., Pohl, K., Goerke, C. \& Wolz, C. Diversification of clonal complex 5 methicillin-resistant Staphylococcus aureus strains (Rhine-Hesse clone) within Germany. J. Clin. Microbiol. 51, 212-216 (2013).

29. Larsen, J. et al. Meticillin-resistant Staphylococcus aureus CC398 is an increasing cause of disease in people with no livestock contact in Denmark, 1999 to 2011. Euro Surveill. 20, 30021 (2015)

30. Sieber, R. N. et al. Drivers and dynamics of methicillin-resistant livestockassociated Staphylococcus aureus CC398 in pigs and humans in Denmark. mBio 9, e02142-18 (2018).

31. European Committee for Antimicrobial Susceptibility Testing (EUCAST) of the European Society of Clinical Microbiology and Infectious Diseases (ESCMID). Determination of minimum inhibitory concentrations (MICs) of antibacterial agents by broth dilution. Clin. Microbiol. Infect. 9, ix-xv (2003).

32. Arndt, D. et al. PHASTER: a better, faster version of the PHAST phage search tool. Nucleic Acids Res. 44, W16-W21 (2016).

33. Winstel, V., Sanchez-Carballo, P., Holst, O., Xia, G. \& Peschel, A. Biosynthesis of the unique wall teichoic acid of Staphylococcus aureus lineage ST395. MBio $\mathbf{5}$, e00869 (2014).

34. Tormo, M. A. et al. Staphylococcus aureus pathogenicity island DNA is packaged in particles composed of phage proteins. J. Bacteriol. 190, 2434-2440 (2008).

35. Chen, P. S., Toribara, T. Y. \& Warner, H. Microdetermination of phosphorus. Anal. Chem. 28, 1756-1758 (1956).

36. Smith, R. L. \& Gilkerson, E. Quantitation of glycosaminoglycan hexosamine using 3-methyl-2-benzothiazolone hydrazone hydrochloride. Anal. Biochem. 98 478-480 (1979).

37. Xia, G. et al. Glycosylation of wall teichoic acid in Staphylococcus aureus by TarM. J. Biol. Chem. 285, 13405-13415 (2010).

38. Brückner, R. A series of shuttle vectors for Bacillus subtilis and Escherichia coli. Gene 122, 187-192 (1992).

39. Liu, H. \& Naismith, J. H. An efficient one-step site-directed deletion, insertion, single and multiple-site plasmid mutagenesis protocol. BMC Biotechnol. 8, 91 (2008).

40. Monk, I. R., Shah, I. M Xu, M., Tan, M. W. \& Foster, T. J. Transforming the untransformable: application of direct transformation to manipulate genetically Staphylococcus aureus and Staphylococcus epidermidis. MBio 3, e00277-11 (2012).

41. Bae, T. \& Schneewind, O. Allelic replacement in Staphylococcus aureus with inducible counter-selection. Plasmid 55, 58-63 (2006).

42. Kabsch, W. Xds. Acta Crystallogr. D Biol. Crystallogr. 66, 125-132 (2010),

43. Sheldrick, G. M. Experimental phasing with SHELXC/D/E: combining chain tracing with density modification. Acta Crystallogr. D Biol. Crystallogr. 66, 479-485 (2010).

44. Vonrhein, C.. Blanc, E., Roversi, P. \& Bricogne, G. Automated structure solution with autoSHARP. Methods Mol. Biol. 364, 215-230 (2007)

45. Adams, P. D. et al. PHENIX: a comprehensive Python-based system for macromolecular structure solution. Acta Crystallogr. D Biol. Crystallogr. 66, 213-221 (2010).

46. Emsley, P., Lohkamp, B., Scott, W. G. \& Cowtan, K. Features and development of Coot. Acta Crystallogr. D Biol. Crystallogr. 66, 486-501 (2010).

47. Murshudov, G. N. et al. REFMAC5 for the refinement of macromolecular crystal structures. Acta Crystallogr. D Biol. Crystallogr. 67, 355-367 (2011).

48. Murshudov, G. N., Vagin, A. A. \& Dodson, E. J. Refinement of macromolecular structures by the maximum-likelihood method. Acta Crystallogr. D Biol. Crystallogr. 53, 240-255 (1997)

49. McCoy, A. J. et al. Phaser crystallographic software. J. Appl. Crystallogr. 40, 658-674 (2007)

50. Schüttelkopf, A. W. \& van Aalten, D. M. PRODRG: a tool for high-throughput crystallography of protein-ligand complexes. Acta Crystallogr. D Biol. Crystallogr. 60, 1355-1363 (2004)

51. Schrodinger, LLC. The PyMOL Molecular Graphics System, Version 1.8 (2015).

52. Chen, V. B. et al. MolProbity: all-atom structure validation for macromolecular crystallography. Acta Crystallogr. D Biol. Crystallogr. 66, 12-21 (2010). 


\section{RESEARCH LETTER}

53. Beaucage, S. L. \& Caruthers, M. H. Deoxynucleoside phosphoramidites-a new class of key intermediates for deoxypolynucleotide synthesis. Tetrahedr. Lett. 22, 1859-1862 (1981).

54. Elie, C. J. J. et al. Synthesis of fragments of the capsular polysaccharide of Haemophilus influenzae type b: Part IIII-3. A solid-phase synthesis of a spacer-containing ribosylribitol phosphate hexamer. Recl. Trav. Chim. Pays Bas 108, 219-223 (1989)

55. Dreef, C. E., Elie, C. J. J., Hoogerhout, P., van der Marel, G. A. \& van Boom, J. H. Synthesis of 1-0-(1,2-di-O-palmitoyl-sn-glycero-3-phospho)-d-myo-inositol 4,5-bisphosphate: an analogue of naturally occurring (ptd) Ins $(4,5) \mathrm{P}_{2}$. Tetrahedr. Lett. 29, 6513-6515 (1988).

56. Dürr, M. C. et al. Neutrophil chemotaxis by pathogen-associated molecular patterns-formylated peptides are crucial but not the sole neutrophil attractants produced by Staphylococcus aureus. Cell. Microbiol. 8, 207-217 (2006).

57. Caulfield, M. J. et al. Small molecule mimetics of an HIV-1 gp41 fusion intermediate as vaccine leads. J. Biol. Chem. 285, 40604-40611 (2010). 
a

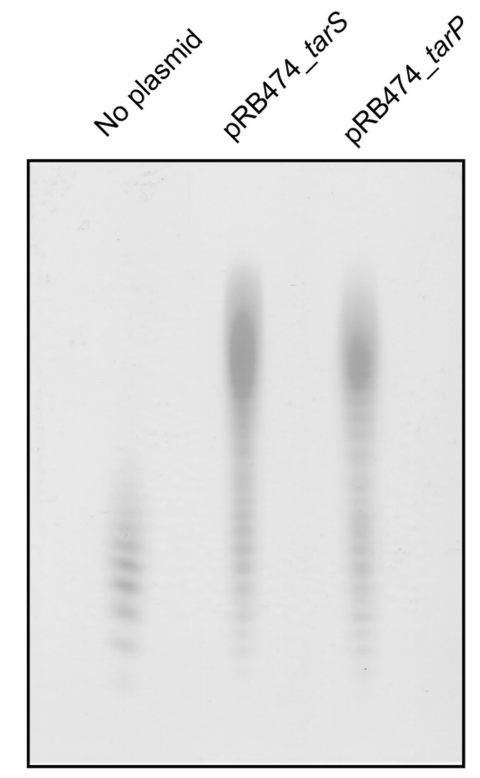

e

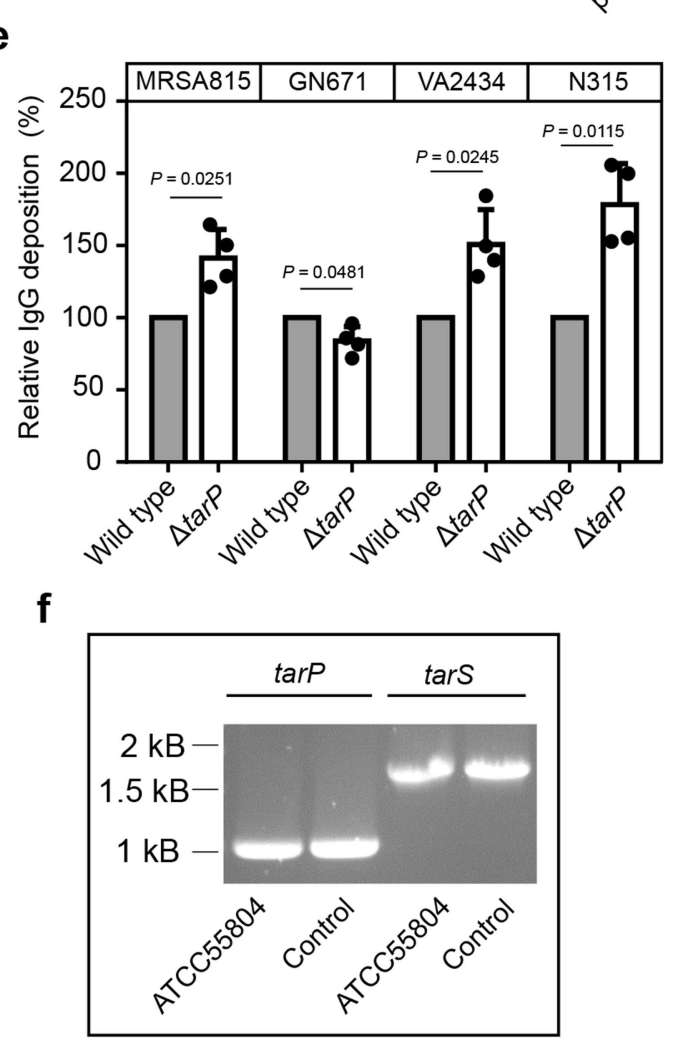

b

\begin{tabular}{|c|c|c|c|}
\hline S. aureus MW2 & Wild type & $\Delta \operatorname{tar} S$ & $\Delta \operatorname{tarS} \mathrm{c}-\operatorname{tar} P$ \\
\hline Plasmid & pRB474 & pRB474 & pRB474_tarP \\
\hline
\end{tabular}

MIC oxacillin $(\mu \mathrm{gg} / \mathrm{ml}) \quad 64 \quad 6 \quad 64$

C
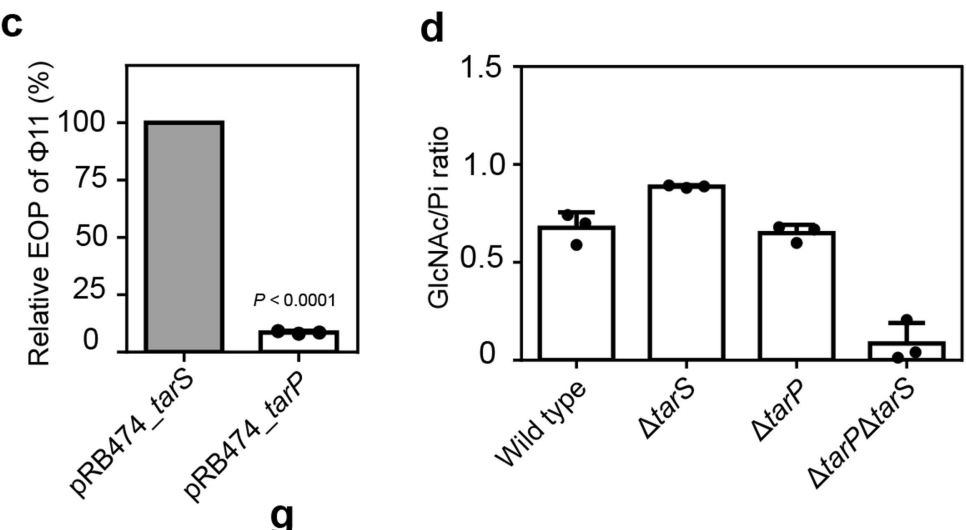

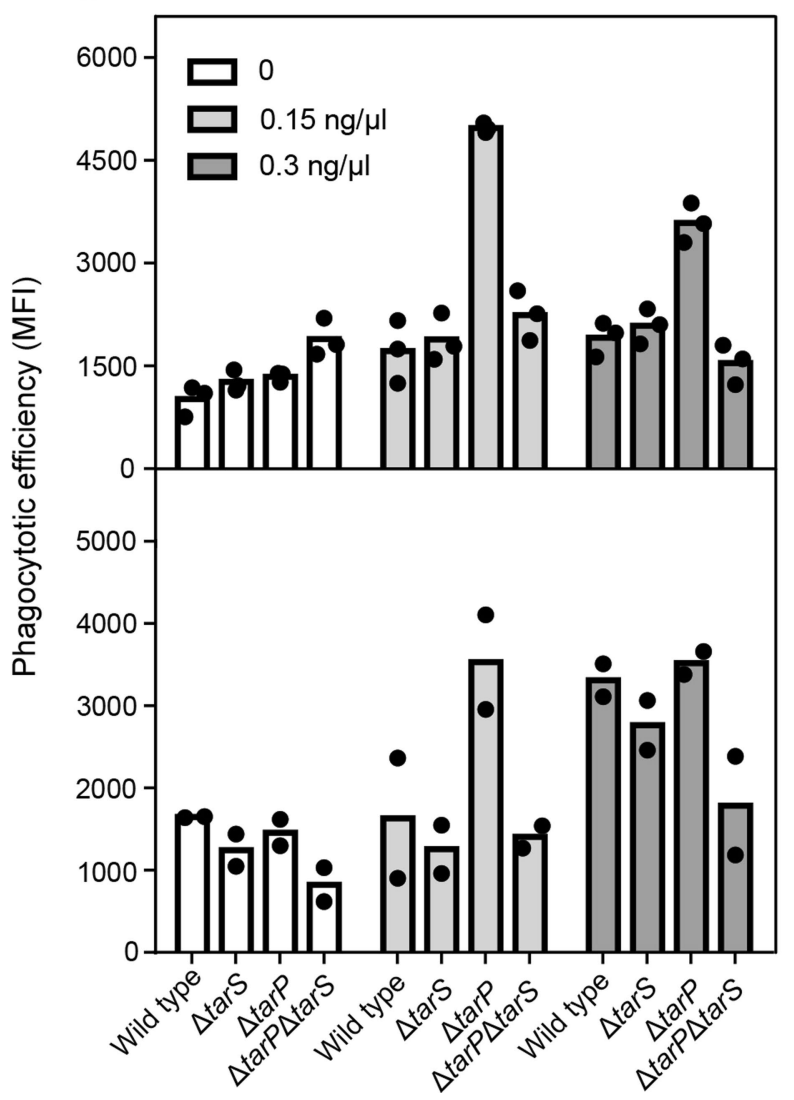

Extended Data Fig. 1 | See next page for caption. 
Extended Data Fig. 1 | Characterization of TarP, deposition of human IgGs, and presence of $\operatorname{tar} P$ in the producer of antigen 336. a, Analysis of WTA by PAGE. WTA from RN4220 $\Delta$ tarM/S expressing either $\operatorname{tar} P$ or tarS was compared with non-glycosylated WTA. Data shown are representative of two experiments. b, MIC values of oxacillin against MW2 wild type, tarS mutant, and $\operatorname{tar} P$-complemented tarS mutant. Data are medians of ten independent experiments. c, Efficiency of plating (EOP) of phage $\Phi 11$ against tarS or tarP-expressing RN4420 $\Delta$ tarM/S. Values of $\operatorname{tar} P$ relative to $\operatorname{tar} S$ expression are given as mean \pm s.d. $(n=3)$. Statistical significance was calculated by paired Student's $t$-test (twosided) with significant $P$ values $(P \leq 0.05)$ indicated. d, The level of WTA glycosylation catalysed by TarP or TarS was determined by analysing the GlcNAc and phosphate content of WTA isolated from a N315 strain panel. Depicted is the ratio of GlcNAc and phosphate as mean with s.d. of three technical replicates. The values are in good agreement with NMR data
(Supplementary Table 3). e, Relative deposition of IgG from intravenous immunoglobulins enriched for WTA binding on different CC5 wildtype and $\operatorname{tar} P$ mutant cells. Values are given as mean percentage \pm s.d. of four independent experiments. Statistical significance was calculated by paired Student's $t$-test (two-sided). $P$ values $\leq 0.05$ were considered significant and are indicated. f, Presence of $\operatorname{tar} P$ and $\operatorname{tar} S$ in S. aureus ATCC55804, expressing antigen 336, described as 3-O-GlcNAc-WTA ${ }^{25}$. Shown is a representative of two independent replicates. $g$, TarP reduces neutrophil phagocytosis of N315 strains lacking protein A, opsonized with indicated concentrations of IgG enriched for WTA binding. Values are depicted as mean fluorescence intensity (MFI). Shown are two independent experiments with neutrophils from different donors. These data supplement data presented in Fig. 4b: upper panel, mean of three technical replicates of an independent experiment, lower panel, mean of two technical replicates. 

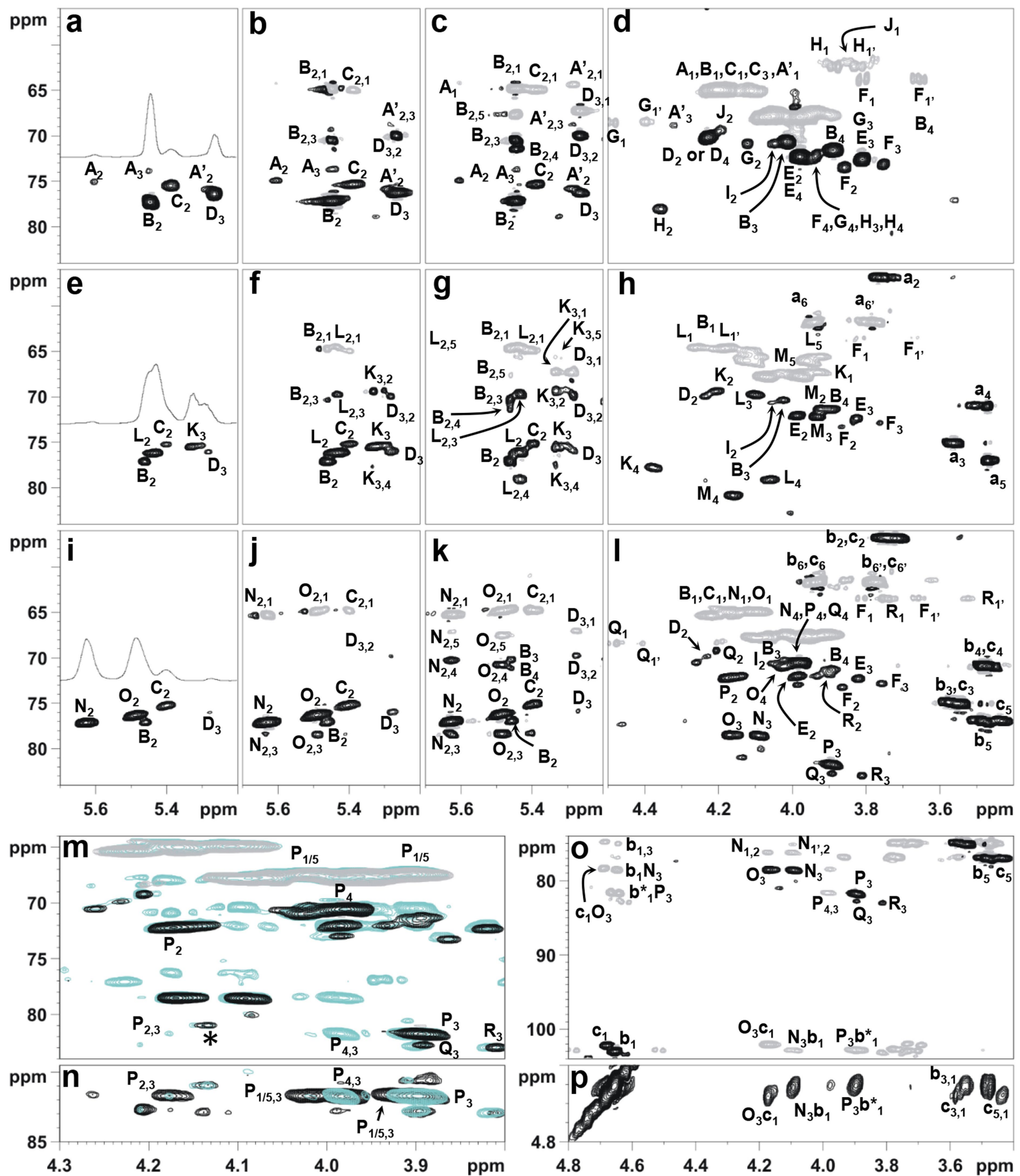

Extended Data Fig. 2 | NMR analysis of WTA from N315 mutant panel. All depicted experiments were repeated twice. $y$-axes and $x$-axes show ${ }^{13} \mathrm{C}$ and ${ }^{1} \mathrm{H}$ chemical shifts, respectively. a-d, NMR spectra of nonglycosylated WTA ( $\Delta \operatorname{tar} S \Delta \operatorname{tar} P$ mutant). a, HSQC expansion of the region containing the ribitol and glycerol protons shifted by acylation; b, c, HSQC-TOCSY-20 and HSQC-TOCSY-80 spectra, respectively. d, HSQC area of the non-acylated ribitol and glycerol proton. $\mathbf{e}-\mathbf{h}, \mathrm{NMR}$ spectra of TarS-WTA ( $\triangle$ tarP mutant). e, HSQC expansion of the region containing the ribitol and glycerol protons shifted by acylation.

f, g, HSQC-TOCSY-20 and HSQC-TOCSY-80, respectively. h, HSQC area of the non-acylated ribitol and glycerol proton. $\mathbf{i}-\mathbf{o}$, NMR spectra of TarPWTA ( $\Delta$ tarS mutant). i, HSQC expansion of the region containing the

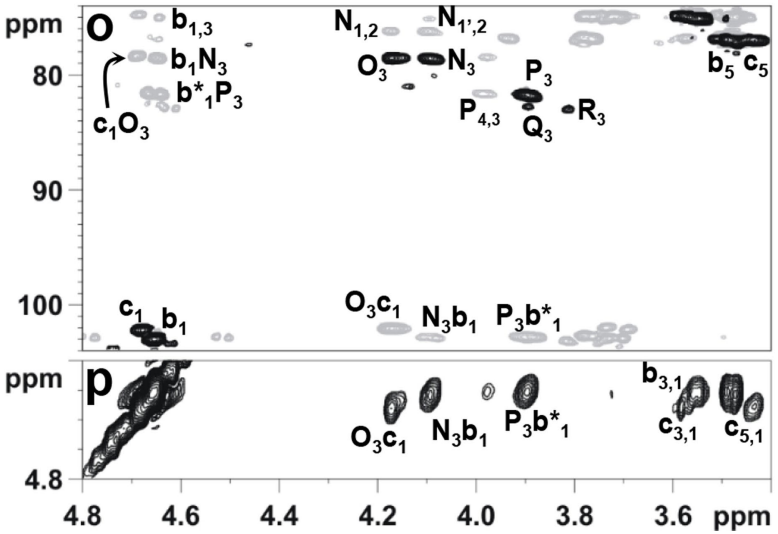

ribitol and glycerol protons shifted by acylation. j, k, HSQC-TOCSY-20 and HSQC-TOCSY-80 spectra, respectively. 1, HSQC area of the nonacylated ribitol and glycerol protons. $\mathbf{m}$, Expansion of $\mathbf{l}$ with HSQC (black/grey) overlapped with HSQC-TOCSY-20 (cyan). n, Overlap of HSQC-TOCSY-20 (cyan) and HSQC-TOCSY-80 (black). o, HSQC (black) and HMBC (grey) detailing the GlcNAc signals. p, NOESY expansion detailing the correlations of the $\beta$-GlcNAc anomeric protons: GlcNAc ' $b$ *' differs from unit ' $b$ ', which has the same anomeric proton chemical shift, but is linked to a different ribitol unit. All densities are labelled with the letters used in Supplementary Table 2. The density marked with an asterisk in $\mathbf{m}$ is consistent with ribitol glycosylated at $\mathrm{O}-4$. 


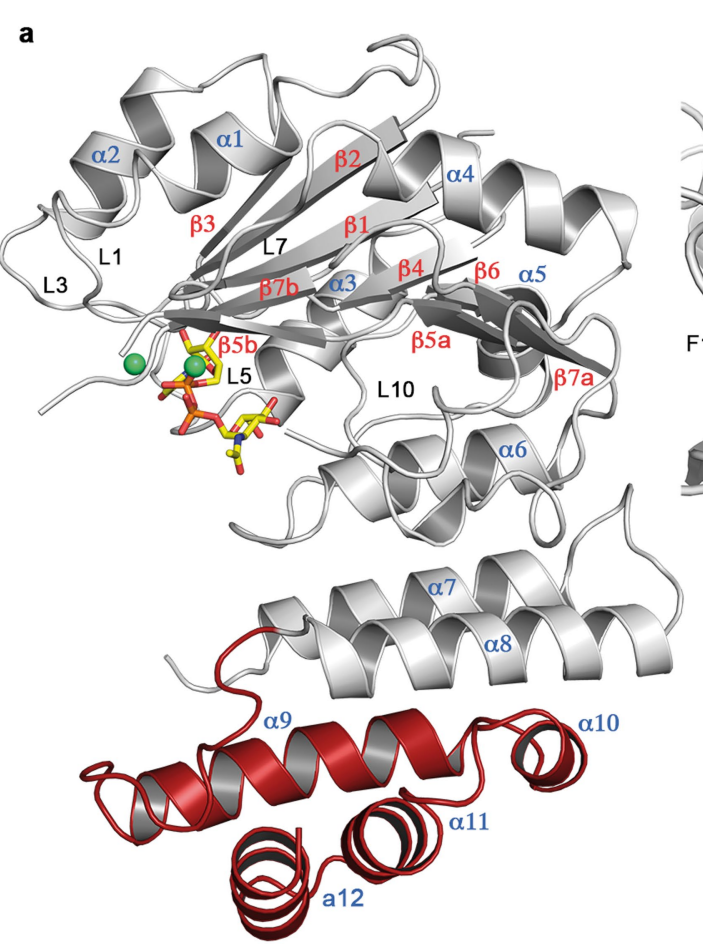

Extended Data Fig. 3 | Secondary structure of a TarP monomer and interactions with UDP-GIcNAc. a, Cartoon representation of a TarP monomer bound to UDP-GlcNAc (yellow) and $\mathrm{Mn}^{2+}$ (lime green). The CTD is coloured red. b. Interactions of TarP with UDP-GlcNAc and $\mathrm{Mn}^{2+}$, coloured as in a. Hydrogen bonds and salt bridges are shown as black dashed lines. c, Interactions of TarP with UDP-GlcNAc (yellow) and $\mathrm{Mg}^{2+}$ (magenta). d, Simulated-annealing $\left(\mathrm{m} F_{\mathrm{o}}-\mathrm{D} F_{\mathrm{c}}\right)$ omit map of b
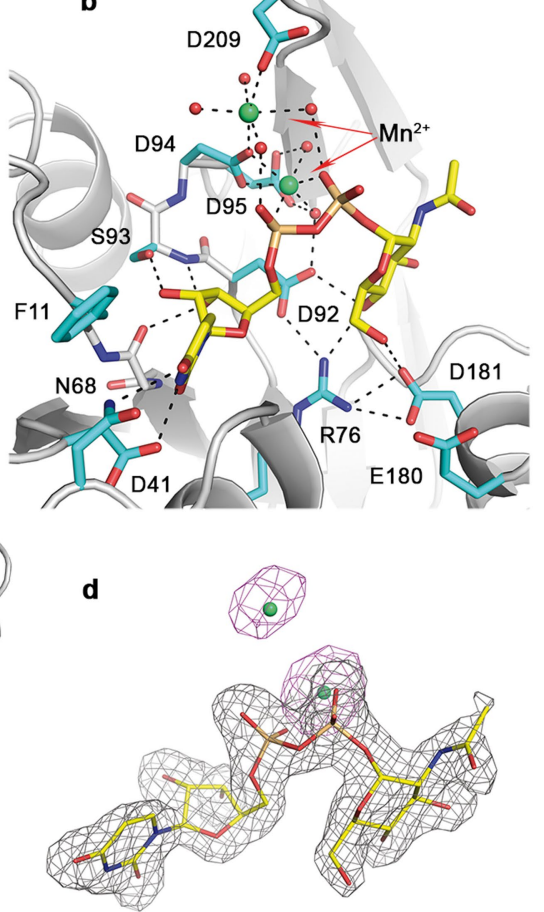
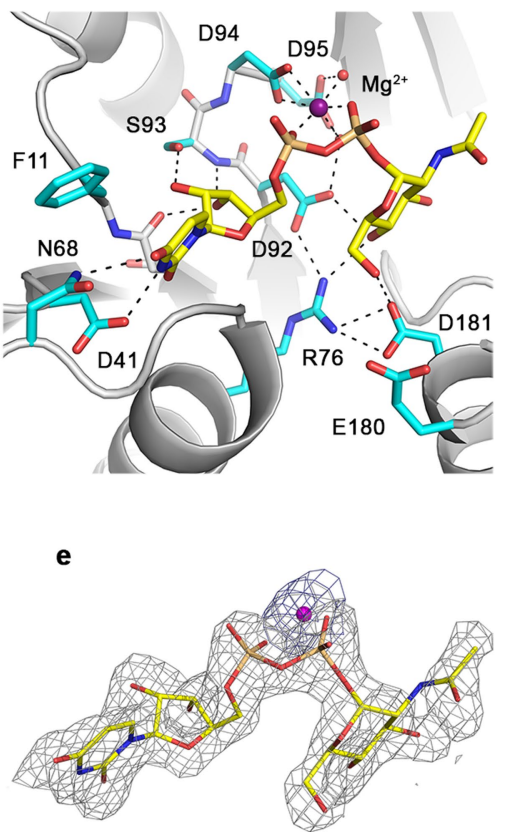

UDP-GlcNAc (grey mesh, contoured at $2.0 \sigma$ ) and $\mathrm{Mn}^{2+}$ (magenta mesh, at $3.0 \sigma$ ) in the TarP-UDP-GlcNAc- $\mathrm{Mn}^{2+}$ complex structure. UDP-GlcNAc and $\mathrm{Mn}^{2+}$ are coloured as in a. e, Simulated-annealing $\left(\mathrm{m} F_{\mathrm{o}}-\mathrm{D} F_{\mathrm{c}}\right)$ omit map of UDP-GlcNAc (grey mesh, at $2.0 \sigma$ ) and $\mathrm{Mg}^{2+}$ (blue mesh, at $2.0 \sigma$ ) in the TarP-UDP-GlcNAc- $\mathrm{Mg}^{2+}$ complex structure. UDP-GlcNAc and $\mathrm{Mg}^{2+}$ are coloured as in $\mathbf{c}$. 
a<smiles>O=P([O-])(O)OC[C@H](O)[C@@H](O)[C@@H](O)COP(=O)([O-])OC[C@H](O)[C@@H](O)[C@H](O)COP(=O)([O-])OC[C@H](O)[C@H](O)[C@H](O)CO</smiles><smiles>CC(CCCCCCN)OP(=O)([O-])OC[C@H](O)[C@H](O)[C@H](O)CO</smiles>

b

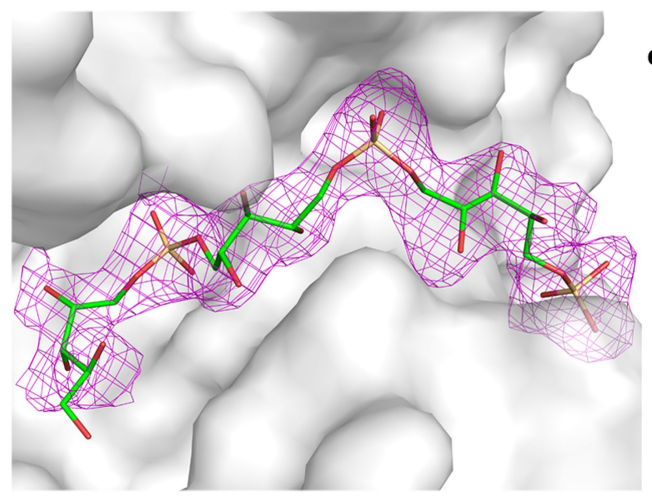

d

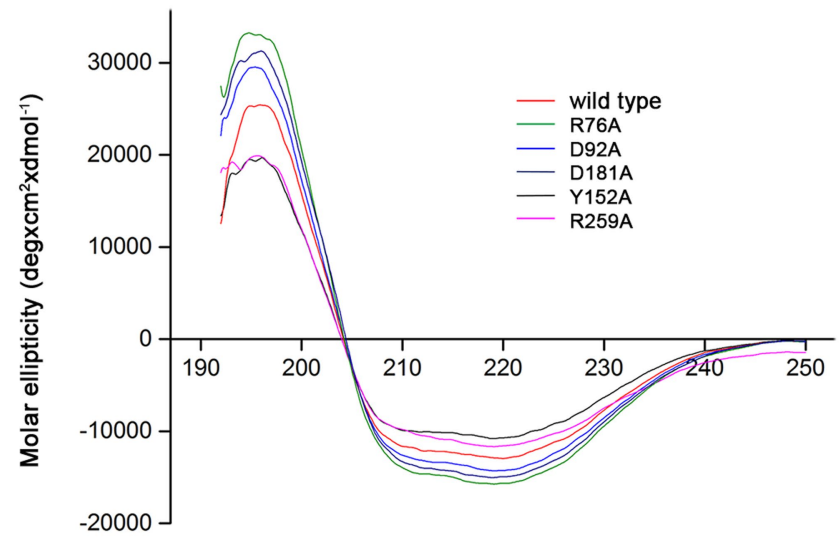

Wavelength $(\mathrm{nm})$

Extended Data Fig. $4 \mid$ Simulated-annealing $\left(\mathrm{m} F_{\mathrm{o}}-\mathrm{D} F_{\mathrm{c}}\right)$ omit maps of 3RboP and UDP-GlcNAc, and characterization of TarP mutant proteins. a, Chemical structures of synthetic $3 \mathrm{RboP}$ and $6 \mathrm{RboP}$ -

$\left(\mathrm{CH}_{2}\right)_{6} \mathrm{NH}_{2}$. The unit numbers are indicated. b. Simulated-annealing $\left(\mathrm{m} F_{\mathrm{o}}-\mathrm{D} F_{\mathrm{c}}\right)$ omit map of $3 \mathrm{RboP}$ (lime green) in the binary structure (magenta mesh, contoured at $2.0 \sigma)$. c, Simulated-annealing $\left(\mathrm{m} F_{\mathrm{o}}-\mathrm{D} F_{\mathrm{c}}\right)$ omit map of UDP-GlcNAc (yellow), $\mathrm{Mg}^{2+}$ (magenta) and 3RboP (lime green) in the ternary complex structure (red mesh, at $1.8 \sigma$, blue mesh,

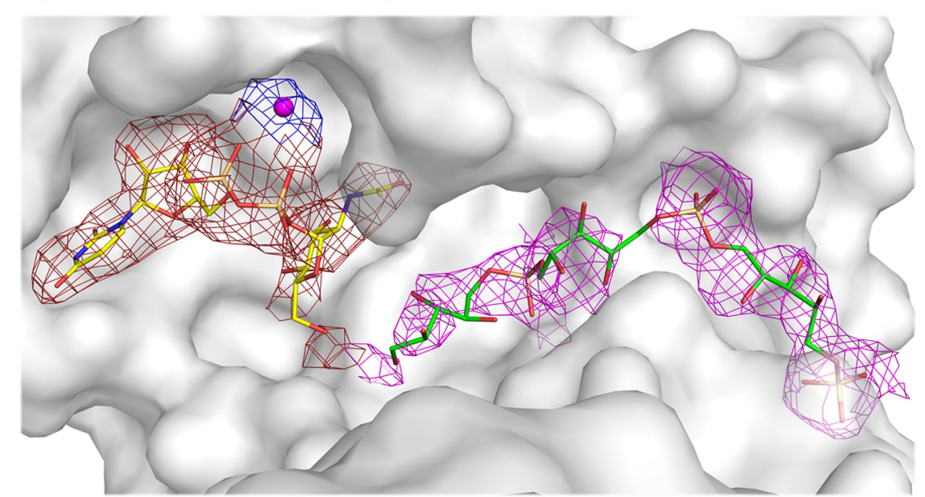

e

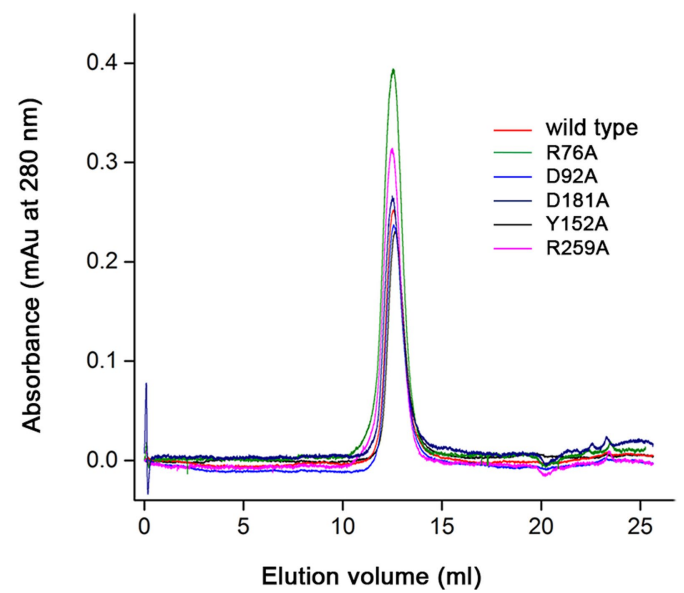

at $2.0 \sigma$ or magenta mesh, at $1.5 \sigma)$. d, Circular dichroism spectra of wildtype and mutant TarP proteins (for wild type, R76A and D181A, $n=3$; for D92A, Y152A and R259A, $n=2$ ). e, Size-exclusion chromatography elution profiles of wild-type and mutant TarP proteins (for wild type, $n=8$; for R76A, D181A and R259A, $n=3$; for D92A and Y152A, $n=2$, all with similar results). Mutant proteins D94A, E180A, D209A, K255A, R262A, and H263A showed similar circular dichroism spectra and sizeexclusion chromatography elution profiles (data not shown). 
a

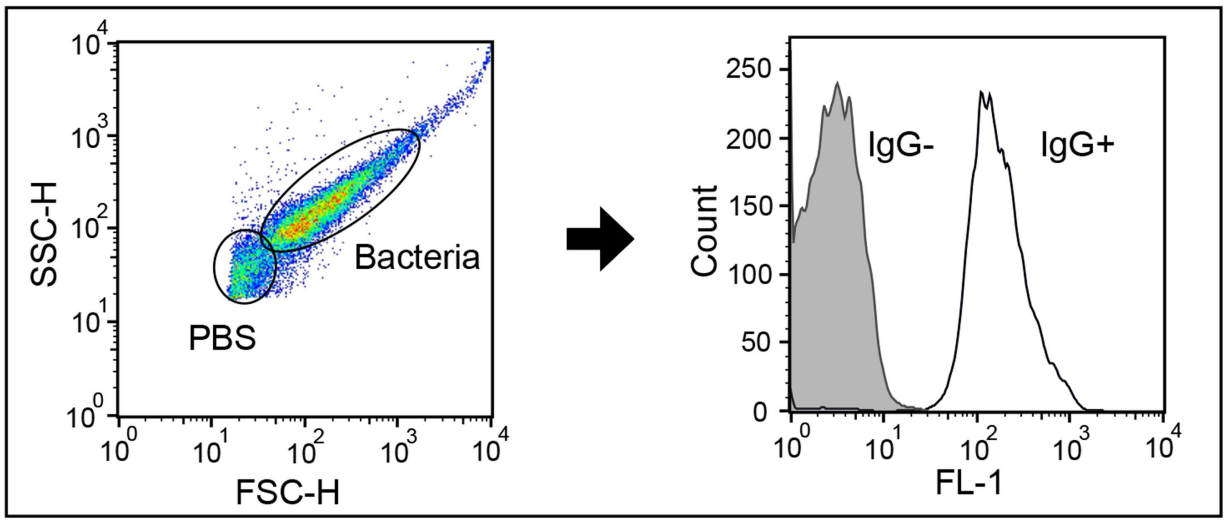

b

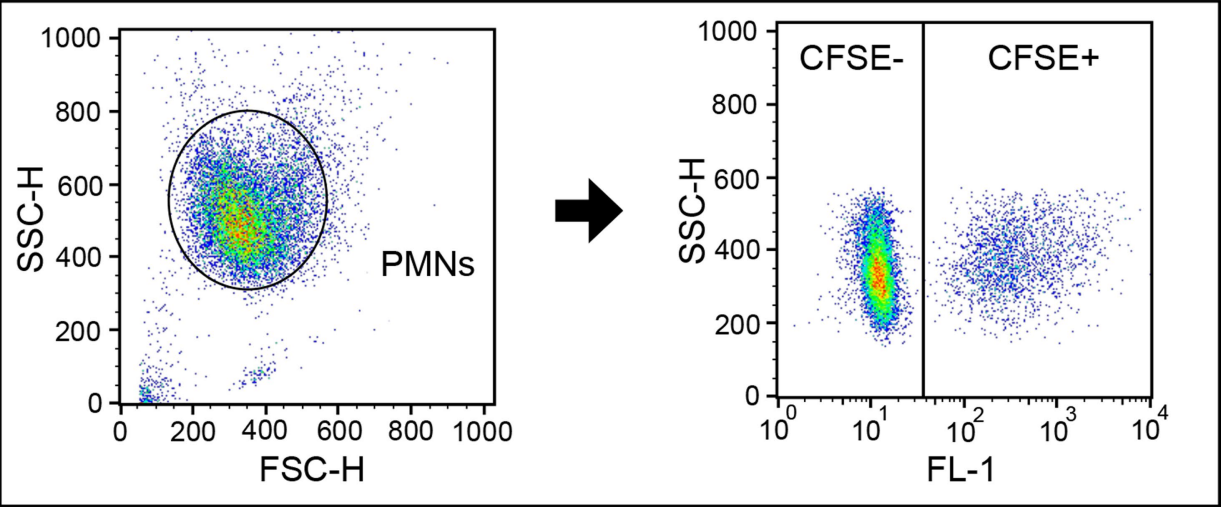

Extended Data Fig. 5 | Gating strategy for flow cytometry experiments. a, Gating strategy for IgG deposition experiments. To distinguish bacteria from background signals, pure PBS was measured. Left, bacterial gating occurred at the FSC/SCC density plot omitting PBS-derived signals. Bacterial aggregates of high SSC and FSC values were excluded from the gated population as well. Right, the mean fluorescence of the bacterial population (black) was determined and compared with non-IgG-treated bacteria (grey) to control for nonspecific binding of the secondary FITClabelled antibody. Subsequently, mean fluorescence values of individual mutants were compared relatively to the corresponding wild-type strain. b, Gating strategy for phagocytosis experiments. Neutrophils were separated by Histopaque/Ficoll gradient and subsequent gating of neutrophils occurred at the FSC/SCC density plot upon size and complexity (left). Histopaque/Ficoll gradient isolations showed a neutrophil purity of more than $80 \%$. Using the CFSE-fluorescence channel, the gated population was subdivided into fluorescence-positive and -negative cells (right). Successful phagocytosis was indicated by uptake of CFSE-labelled bacteria. The phagocytic efficiency was expressed as product of the mean fluorescence of the fluorescence-positive population and their relative abundance (mean fluorescence intensity, MFI). 
Extended Data Table 1 | tarP presence and podophage susceptibility of CC5 strains, comprising sequence type (ST) 5 and 225 , and CC398 isolates

\begin{tabular}{|c|c|c|c|c|c|c|}
\hline Collection & \multicolumn{2}{|c|}{ Rhine-Hesse collection } & \multicolumn{2}{|c|}{ Danish LA-MRSA collection } & \multicolumn{2}{|c|}{ MRSA collection Tübingen } \\
\hline Clonal complex & $5(\mathrm{ST} 5+\mathrm{ST})$ & & 398 & & 5 (ST5 + ST & \\
\hline tarP status & Negative & Positive & Negative & Positive & Negative & Positive \\
\hline $\mathrm{n}$ & 21 & 39 & 18 & 12 & 11 & 53 \\
\hline Phage susceptibility & Susceptible & Resistant & Susceptible & Resistant & Susceptible & Resistant \\
\hline$\phi 44$ & 21 & 39 & 18 & 12 & ND & ND \\
\hline$\phi 66$ & 21 & 39 & 18 & 12 & ND & ND \\
\hline$\phi \mathrm{P} 68$ & 21 & 39 & 18 & 12 & ND & ND \\
\hline
\end{tabular}

tarP presence in three different S. aureus collections was determined by PCR using primer pair TarP_Ty_Fw/Rv. Phage susceptibility to podophages $\Phi 44, \Phi 66$, and $\Phi P 68$ was determined by soft-agar overlay. Plaque formation indicated susceptibility, absence of visible plaque formation indicated resistance. ND, not determined. 
RESEARCH LETTER

Extended Data Table 2 | Enzymatic activities of mutated TarP proteins

\begin{tabular}{lll}
\hline Function & TarP variant & Activity of wild type in \% \\
\hline Trimer interface & I322E & 128 \\
\hline \multirow{2}{*}{ UDP-GIcNAc binding } & R76A & 1 \\
& D92A & 2 \\
& D94A & 14 \\
D209A & 105 \\
\hline Catalytic base & E180A & 15 \\
\hline & D181A & 1 \\
\hline \multirow{3}{*}{ 3RboP binding } & Y152A & 44 \\
& K255A & 99 \\
& R259A & 3 \\
& R262A & 97 \\
& H263A & 81 \\
\hline
\end{tabular}


Extended Data Table 3 | Donor substrate specificity of TarP

\begin{tabular}{ll}
\hline Sugar nucleotide & Enzymatic activity $\left(\mathrm{nmol} / \mathrm{mg}^{*} \mathrm{~min}\right)$ \\
\hline UDP-GIcNAc & 2.20 \\
UDP-Glc & 0.01 \\
UDP-GalNAc & 0.03 \\
UDP-Gal & 0.01 \\
\hline
\end{tabular}




\section{RESEARCH LETTER}

Extended Data Table 4 | Crystallographic data statistics for TarP and TarP-UDP-GIcNAc-Mg ${ }^{2+}$

\begin{tabular}{|c|c|c|c|c|}
\hline & TarP native & TarP-SeMet & TarP-SeMet & TarP-UDP-GlcNAc-Mg ${ }^{2+}$ \\
\hline Data collection & & Peak & Inflection & \\
\hline Space group & $\mathrm{P} 2_{1}$ & $\mathrm{P} 2{ }_{1}$ & $\mathrm{P} 2_{1}$ & $\mathrm{P} 2{ }_{1}$ \\
\hline \multicolumn{5}{|l|}{ Cell dimensions } \\
\hline$a, b, c(\AA)$ & $43.37,95.25,125.47$ & $44.06,95.33,130.72$ & $43.99,95.22,130.52$ & $43.85,95.27,130.22$ \\
\hline$\alpha, \beta, y\left(^{\circ}\right)$ & $90.00,96.57,90.00$ & $90.00,93.41,90.00$ & $90.00,93.34,90.00$ & $90.00,93.49,90.00$ \\
\hline Wavelength $(\AA)$ & 1.00004 & 0.97941 & 0.97952 & 0.91841 \\
\hline Resolution $(\AA)$ & $44.5-1.86(1.91-1.86)$ & $47.7-2.29(2.35-2.29)$ & $47.7-2.30(2.35-2.30)$ & $47.6-1.95(2.00-1.95)$ \\
\hline$R_{\text {sym or }} R_{\text {merge }}(\%)$ & $8.4(87.7)$ & $11.5(103.8)$ & $9.7(62.2)$ & $12.6(110.1)$ \\
\hline$I / \sigma(I)^{*}$ & $9.4(1.4)$ & $13.8(1.8)$ & $15.8(2.9)$ & $9.2(1.3)$ \\
\hline $\mathrm{CC}_{1 / 2}(\%)$ & $99.7(50.0)$ & $99.8(64.0)$ & $99.8(81.9)$ & $99.6(50.6)$ \\
\hline Completeness (\%) & $98.5(97.5)$ & $99.0(88.4)$ & $99.2(90.9)$ & $99.9(99.7)$ \\
\hline Redundancy & $2.9(2.7)$ & $7.0(6.5)$ & $6.6(6.0)$ & $5.0(5.0)$ \\
\hline \multicolumn{5}{|l|}{ Phasing } \\
\hline Rcullis (ano) & & 0.76 & & \\
\hline Phasing power & & 1.24 & & \\
\hline HA sites / ASU & & 26 & & \\
\hline FOMacentric & & 0.41 & & \\
\hline \multicolumn{5}{|l|}{ Refinement } \\
\hline Resolution $(\AA)$ & $44.5-1.86$ & & & $47.6-1.95$ \\
\hline No. reflections & $241855(16740)$ & & & $386853(28878)$ \\
\hline$R_{\text {work }} / R_{\text {free }}(\%)$ & $17.1 / 21.8$ & & & $17.7 / 22.4$ \\
\hline \multicolumn{5}{|l|}{ No. atoms } \\
\hline Protein & 7538 & & & 7479 \\
\hline Substrates & 0 & & & 117 \\
\hline lons & 13 & & & 29 \\
\hline Other molecules & 0 & & & 24 \\
\hline Water & 697 & & & 804 \\
\hline \multicolumn{5}{|l|}{ Average $B$-factors $\left(\AA^{2}\right)$} \\
\hline Protein & 31.7 & & & 35.5 \\
\hline Substrates & & & & 43.9 \\
\hline lons & 40.1 & & & 44.6 \\
\hline Other molecules & & & & 39.2 \\
\hline Water & 41.6 & & & 41.0 \\
\hline \multicolumn{5}{|l|}{ R.m.s deviations ${ }^{* *}$} \\
\hline Bond lengths $(\AA)$ & 0.010 & & & 0.008 \\
\hline Bond angles $\left({ }^{\circ}\right)$ & 1.310 & & & 1.254 \\
\hline Allowed (\%) & 3 & & & 3 \\
\hline Outliers (\%) & 0 & & & 0 \\
\hline
\end{tabular}

Values in parentheses are for highest-resolution shell. Two data sets for TarP-SeMet were collected from the same single crystal.

$* I$ is the mean intensity, $\sigma(l)$ is the standard deviation of reflection intensity $I$.

**r.m.s.d., root-mean-square deviation of bond length or bond angle. 
Extended Data Table 5 | Crystallographic data statistics for TarP-UDP-GIcNAc-Mn' ${ }^{2}$, TarP-3RboP, TarP-6RboP-( $\left.\mathrm{CH}_{2}\right)_{6} \mathrm{NH}_{2}$ and TarP-UDP-GIcNAc-3RboP

\begin{tabular}{|c|c|c|c|c|}
\hline & TarP-UDP-GlcNAc-Mn ${ }^{2+}$ & TarP-3RboP & TarP-6RboP-( $\left(\mathrm{CH}_{2}\right)_{6} \mathrm{NH}_{2}$ & TarP-UDP-GlcNAc-3RboP \\
\hline \multicolumn{5}{|l|}{ Data collection } \\
\hline Space group & $\mathrm{P} 2{ }_{1}$ & $\mathrm{P} 2{ }_{1}$ & $\mathrm{P} 2{ }_{1}$ & $\mathrm{P} 2{ }_{1}$ \\
\hline \multicolumn{5}{|l|}{ Cell dimensions } \\
\hline$a, b, c(\AA)$ & $43.86,95.36,130.55$ & $95.61,217.27,123.99$ & $95.41,211.25,122.68$ & $95.17,210.75,123.20$ \\
\hline$\alpha, \beta, y\left(^{\circ}\right)$ & $90.00,93.51,90.00$ & $90.00,91.38,90.00$ & $90.00,91.61,90.00$ & $90.00,91.92,90.00$ \\
\hline Wavelength $(\AA)$ & 0.91840 & 1.00000 & 1.00002 & 1.00002 \\
\hline Resolution $(\AA)$ & $47.7-1.80(1.85-1.80)$ & $49.8-2.16(2.22-2.18)$ & $48.5-2.40(2.46-2.40)$ & $48.4-2.73(2.80-2.73)$ \\
\hline$R_{\text {sym }}$ or $R_{\text {merge }}(\%)$ & $5.6(101.0)$ & $13.7(140.9)$ & $15.6(141.2)$ & $25.4(161.1)$ \\
\hline$I / \sigma(I)^{*}$ & $12.0(1.3)^{\prime}$ & $11.9(1.5)$ & $10.8(1.5)$ & $8.4(1.4)$ \\
\hline $\mathrm{CC}_{1 / 2}(\%)$ & $99.9(51.1)$ & $99.8(54.0)$ & $99.6(50.7)$ & $99.0(52.3)$ \\
\hline Completeness (\%) & $99.8(99.5)$ & $100.0(100.0)$ & $99.9(100.0)$ & $99.9(99.8)$ \\
\hline Redundancy & $3.6(3.3)$ & $7.0(6.6)$ & $6.2(6.4)$ & $7.1(7.4)$ \\
\hline \multicolumn{5}{|l|}{ Refinement } \\
\hline Resolution $(\AA)$ & $47.7-1.80$ & $49.8-2.18$ & $48.5-2.40$ & $48.4-2.73$ \\
\hline No. reflections & 355981 (24195) & $1833608(128618)$ & 1172903 (89756) & 911354 (69899) \\
\hline$R_{\text {work }} / R_{\text {free }}(\%)$ & $17.6 / 21.3$ & $17.1 / 20.7$ & $19.6 / 22.7$ & $19.2 / 23.5$ \\
\hline \multicolumn{5}{|l|}{ No. atoms } \\
\hline Protein & 7,543 & 29,987 & 29,709 & 29,439 \\
\hline Substrates & 117 & 480 & 480 & 948 \\
\hline lons & 19 & 32 & 16 & 35 \\
\hline Other molecules & 12 & 18 & & \\
\hline Water & 739 & 2,694 & 1,555 & 1,383 \\
\hline \multicolumn{5}{|c|}{ Average $B$-factors $\left(\AA^{2}\right)$} \\
\hline Protein & 37.6 & 46.1 & 51.2 & 53.0 \\
\hline Substrates & 38.4 & 57.8 & 75.0 & 84.3 \\
\hline lons & 47.4 & 52.7 & 54.0 & 50.6 \\
\hline Other molecules & 46.6 & 49.7 & & \\
\hline Water & 43.7 & 49.4 & 48.6 & 41.4 \\
\hline \multicolumn{5}{|l|}{ R.m.s deviations ${ }^{* *}$} \\
\hline Bond lengths $(\AA)$ & 0.010 & 0.009 & 0.008 & 0.010 \\
\hline Bond angles $\left({ }^{\circ}\right)$ & 1.331 & 1.288 & 1.214 & 1.302 \\
\hline \multicolumn{5}{|l|}{ Ramachandran plot } \\
\hline Favored (\%) & 98.0 & 97.0 & 96.8 & 96.4 \\
\hline Allowed (\%) & 2.0 & 3.0 & 3.2 & 3.6 \\
\hline Outliers (\%) & 0 & 0 & 0 & 0 \\
\hline
\end{tabular}

Values in parentheses are for highest-resolution shell.

$* l$ is the mean intensity, $\sigma(l)$ is the standard deviation of reflection intensity $I$.

**r.m.s.d., root-mean-square deviation of bond length or bond angle. 


\section{Reporting Summary}

Nature Research wishes to improve the reproducibility of the work that we publish. This form provides structure for consistency and transparency in reporting. For further information on Nature Research policies, see Authors \& Referees and the Editorial Policy Checklist.

\section{Statistical parameters}

When statistical analyses are reported, confirm that the following items are present in the relevant location (e.g. figure legend, table legend, main text, or Methods section).
n/a Confirmed
$\square \bigotimes$ The exact sample size $(n)$ for each experimental group/condition, given as a discrete number and unit of measurement
$\square$ \ An indication of whether measurements were taken from distinct samples or whether the same sample was measured repeatedly
$\square \bigotimes \begin{aligned} & \text { The statistical test(s) used AND whether they are one- or two-sided } \\ & \text { Only common tests should be described solely by name; describe more complex techniques in the Methods section. }\end{aligned}$
Х $\square$ A description of all covariates tested
Х $\square$ A description of any assumptions or corrections, such as tests of normality and adjustment for multiple comparisons
$\square \bigotimes \begin{aligned} & \text { A full description of the statistics including central tendency (e.g. means) or other basic estimates (e.g. regression coefficient) AND } \\ & \text { variation (e.g. standard deviation) or associated estimates of uncertainty (e.g. confidence intervals) }\end{aligned}$
$\square \bigotimes \begin{aligned} & \text { For null hypothesis testing, the test statistic (e.g. } F, t, r \text { ) with confidence intervals, effect sizes, degrees of freedom and } P \text { value noted } \\ & \text { Give } P \text { values as exact values whenever suitable. }\end{aligned}$
Х $\square$ For Bayesian analysis, information on the choice of priors and Markov chain Monte Carlo settings
Х $\square$ For hierarchical and complex designs, identification of the appropriate level for tests and full reporting of outcomes
$\bigotimes \square$ Estimates of effect sizes (e.g. Cohen's $d$, Pearson's $r$ ), indicating how they were calculated
$\varnothing$ Clearly defined error bars
State explicitly what error bars represent (e.g. SD, SE, CI)

Our web collection on statistics for biologists may be useful.

\section{Software and code}

Policy information about availability of computer code

Data collection - Genomes containing tarP were identified by the BLAST algorithm (URL: https://blast.ncbi.nlm.nih.gov/Blast.cgi), by using the amino acid sequence of TarP of S. aureus N315 (gene SA1808) as query.

DA+ GUI, DA+ Server, Diffraction Viewer - Albula (version 3.2.0), and Data Processing Tracker were used for data collection of TarP structures at the beamline X06DA of Swiss Light Source in Villigen, Switzerland and Beamline-Control GUI (MXCuBE version 1) was used at the beamline BL14.1 in BESSY-II, Helmholtz Zentrum Berlin. For data reducing, phasing, model building, and refinement XDS package (BUTLT=20160617), XSCALE (BUTLT=20131111), SHELX C/D/E (2013/2), SHARP (2.8.6)/autoSHARP (3.10.6), PHENIX (1.13-2998), COOT (0.8.9.1 EL (CCP4)), REFMAC (CCPInterface 7.0.045), PHASER (CCP4Inetrface 7.0.044), FFT (CCP4Interface 7.0.052), CCP4 (7.0.062), PRODRG (CCP4Interface 7.0.062) were used. The structural figures were generated using PyMOL (1.8.4.1) and the models were valuated using MolProbity (4.02b-467).

- NMR spectra were acquired, processed and analyzed by using Bruker TopSpin 3.1 software for WTA elucidation. For synthesis of chemical compounds Bruker TopSpin 2.1 was used.

Data analysis

-tarP-containing genomes were analyzed by the web-tool PHASTER (URL: http://phaster.ca/).

-Statistical analysis was performed by using GraphPad Prism (GraphPad 419 Software, Inc.) Version 6.

- NMR spectra of WTA were analyzed by using Bruker TopSpin 3.1 software

-Data analysis of NMR of chemical compounds MestReNova; Mnova 12.0.3 was used

For manuscripts utilizing custom algorithms or software that are central to the research but not yet described in published literature, software must be made available to editors/reviewers upon request. We strongly encourage code deposition in a community repository (e.g. GitHub). See the Nature Research guidelines for submitting code \& software for further information. 
Policy information about availability of data

All manuscripts must include a data availability statement. This statement should provide the following information, where applicable:

- Accession codes, unique identifiers, or web links for publicly available datasets

- A list of figures that have associated raw data

- A description of any restrictions on data availability

All major data generated or analyzed in this study are included in the article or its supplementary information files. The coordinates and structure factors were deposited in the Protein Data Bank under accession numbers 6H1J,6H21, 6H2N, 6H4F. and 6H4M and 6HNQ. Source data of experiments with animals (Fig 4c, d), and Gel electrophoresis (Extended data Fig. 1f) are provided as source data. All other data relating to this study are available from the corresponding authors on reasonable request.

\section{Field-specific reporting}

Please select the best fit for your research. If you are not sure, read the appropriate sections before making your selection.
$\bigotimes$ Life sciences
Behavioural \& social sciences
Ecological, evolutionary \& environmental sciences

For a reference copy of the document with all sections, see nature.com/authors/policies/Reportingsummary-flat.pdf

\section{Life sciences study design}

All studies must disclose on these points even when the disclosure is negative.

Sample size Sample sizes of in vitro and in vivo experiments were chosen to obtain significant results. The prospective power analysis was performed with appropriate statistical methods.

Data exclusions One data point ( = one mouse) was not included in the alum control of experiment Fig $4 \mathrm{~d}$. The reason for exclusion was an early euthanization of the animal due to a severe abnormal infection. No data points from other in vitro or in vivo experiments were excluded.

Replication Each major experiement was repeated at least three times as stated in the manuscript. In case of neutrophil phagocytosis assays results exhibited the typical strong donor-specific differences but similar trends between bacterial test strains. Therefore, a typical experiment, representative of 3 independet experiments is shown as stated in the figure legend. The other 2 replicates can be found in the Supplementary Information.

Randomization Neutrophils and plasma IgGs were obtained from three independent randomly selected donors to control for donor-specific differences in the prior exposure to $\mathrm{S}$. aureus antigens.

Blinding Blinding was not necessary in our study.

\section{Reporting for specific materials, systems and methods}

Materials \& experimental systems

\begin{tabular}{l|l}
\hline In & Involved in the study \\
$\square$ & $\bigotimes$ Unique biological materials \\
$\square$ & $\square$ Antibodies \\
$\square$ & X Animals and other organisms \\
\hline
\end{tabular}

\begin{tabular}{l|l}
\multicolumn{2}{l}{ Methods } \\
\hline n/a Involved in the study \\
$\square$ ChIP-seq \\
$\square$ & $\square$ Flow cytometry
\end{tabular}

\section{Unique biological materials}

Policy information about availability of materials

Obtaining unique materials Serum IgG was purified from plasma of ramdomly selected individual donors independently of sex or age. Remaining preparations are stored at $-80^{\circ} \mathrm{C}$ and can be obtained upon request. 
Antibodies used

- IgG was isolated from plasma of individual donors via NAb 393 Protein G Spin Kit (ThermoFischer, Article number 89979) - Pooled human IgG preparations from different suppliers (Abcam, Article number ab98981, lot number not available); (Athens Research \& Technology, Article Number 16-16-090707, Lot number IG2015-02)

- IgG enriched for WTA binding, purified from IVIG in Pusan, South Koreas as described previously (Kurokawa et al., 2013 doi: 10.1074/jbc.M113.509893)

- Goat anti-Human IgG secondary antibody FITC conjugate (Thermo Scientific, Article Number 62-8411, Lot Number RH235723, SC242149, and TF268287, Dilution 1:100 in $100 \mu \mathrm{L}$ PBS per reaction, polyclonal)

Validation

- IgG isolated from plasma of idividual donors was checked for purity by SDS PAGE

- Pooled human IgG preparations were validated as stated by the manufacturers. Abcam: "Purity 96\%. IgA and IgM levels are $<1 \%$ of the total IgG content by RID. ab98981 shows a single band corresponding to gamma electrophoretic mobility by Cellulose Acetate Electrophoresis"; Athens Research \& Technology: "Purity Greater than 95\% by SDS-PAGE".

- IgG enriched for WTA binding, purified from IVIG was checked for purity by SDS-PAGE as described previously (Kurokawa et al., 2013 doi: 10.1074/jbc.M113.509893).

\section{Animals and other organisms}

Policy information about studies involving animals; ARRIVE guidelines recommended for reporting animal research $\begin{array}{ll}\text { Laboratory animals } & \begin{array}{l}6 \text { weeks-old wild-type C57BL/6J mice of 50\% male and 50\% female ratio, } 422 \text { purchased from ORIENT BIO (Charles River } \\ \text { Breeding Laboratories in Korea) }\end{array}\end{array}$

Wild animals

The study did not involve wild animals.

Field-collected samples

The study did not involve field-collected animals or organisms.

\section{Human research participants}

\section{Policy information about studies involving human research participants}

Population characteristics Individual donors of serum IgG and neutrophils were healthy volunteers selected randomly irrespective of sex and age. Donors did not suffer from a documented S. aureus infection.

Recruitment

Donors of serum IgG and neutrophils were healthy volunteers selected randomly irrespective of sex. Donors were of 20-50 years, with a majorities of donors younger than 30 years.

\section{Flow Cytometry}

\section{Plots}

Confirm that:

\The axis labels state the marker and fluorochrome used (e.g. CD4-FITC).

\The axis scales are clearly visible. Include numbers along axes only for bottom left plot of group (a 'group' is an analysis of identical markers).

\All plots are contour plots with outliers or pseudocolor plots.

$\bigotimes$ A numerical value for number of cells or percentage (with statistics) is provided.

Methodology

Sample preparation

Instrument

Software

Cell population abundance

Gating strategy
For IgG binding experiments, bacterial cultures were ajusted to an OD600 of 0.5, diluted 1:10 in PBS, and mixed with antibody solution in a ratio of $1: 2$. Samples were fixed with $2 \%$ paraformaldehyde prior to measurement.

BD FACSCalibur Flow Cytometry, model number 342976

CellQuest Pro version 5, FlowJo V10

Neutrophils were isolated by Histopaque-Ficoll-gradient centrifugation and contaminating erythrocytes were lysed prior to analysis. Due to individual donor characteristics the samples contained an average of approximatly 65-70 \% intact neutrophils, which were separated during measurement from cell debris/erythrocytes via FSC/SSC gating.

In the FACS analysis, bacterial samples showed a purity of over 95\%. Impurities were mainly caused by PBS-derived background signal.

Neutrophil and bacteria gating occured at the FSC/SCC density plot. To distingush neutrophils from cell debris neutrophils were gated based on size and complexity. For bacterial cell gating, pure PBS was measured to define the background signal. 95\% of 
this background signal was excluded using a treshold on FSC and SSC. The bacteria were then gated according to their size and complexity.

$\bigotimes$ Tick this box to confirm that a figure exemplifying the gating strategy is provided in the Supplementary Information. 